\title{
Scattering on two Aharonov-Bohm vortices with opposite fluxes
}

\author{
E Bogomolny ${ }^{1}$, S Mashkevich ${ }^{2}$, and S Ouvry ${ }^{1}$ \\ ${ }^{1}$ CNRS, Université Paris-Sud, UMR 8626 \\ Laboratoire de Physique Théorique et Modèles Statistiques, 91405 Orsay, France \\ 2 Schrödinger, 120 West 45th St., New York, NY 10036, USA \\ and Bogolyubov Institute for Theoretical Physics, Kiev 03680, Ukraine \\ E-mail: eugene.bogomolny@lptms.u-psud.fr, mash@mashke.org, \\ stephane.ouvry@lptms.u-psud.fr
}

\begin{abstract}
The scattering of an incident plane wave on two Aharonov-Bohm vortices with opposite fluxes is considered in detail. The presence of the vortices imposes nontrivial boundary conditions for the partial waves on a cut joining the two vortices. These conditions result in an infinite system of equations for scattering amplitudes between incoming and outgoing partial waves, which can be solved numerically. The main focus of the paper is the analytic determination of the scattering amplitude in two limits, the small flux limit and the limit of small vortex separation. In the latter limit the dominant contribution comes from the $S$-wave amplitude. Calculating it, however, still requires solving an infinite system of equations, which is achieved by the Riemann-Hilbert method. The results agree well with the numerical calculations.
\end{abstract}

PACS numbers: 03.65.Vf, 03.65.Ge, 02.30.Rz, 11.55.Ds 


\section{Introduction}

The Aharonov-Bohm problem [1] certainly leads to one of the most fascinating quantum mechanical effects which has been experimentally tested [2].

Clearly the original model as stated in the seminal 1959 paper [1] is by itself somehow an abstraction. Leaving aside the zero flux size limit issue and the related question of the behaviour of wave functions at the location of the vortex (see, e.g., [3]) there is not such a physical system where the flux of a magnetic field can pierce a plane at a certain location without returning at some other place on the plane so that the total flux is zero. In the Aharonov-Bohm paper this issue is solved by assuming that the return flux is spread far away on the plane (in fact at infinity) so that its direct effect on the problem at hand can be neglected. This simplifying assumption is crucial in rendering the model exactly solvable, and most of the research effort has been devoted to this situation (see, e.g., [4], [5] and references therein).

We would like to address here a more realistic situation where the piercing flux returns at a finite distance. More precisely, we consider two vortices of opposite strength piercing the plane at distance $2 R$ one from the other. Doing so, we move from the solvable one-vortex standard Aharonov-Bohm problem to a two-vortex problem, which is more difficult to address, with no explicit solution at disposal.

A few many-vortex Aharonov-Bohm problems have already been considered in the literature, in particular the scattering on vortices arranged on a lattice [6] or vortices whose locations are random [7].

The problem of scattering of an incident plane wave on several vortices has mostly been discussed by two methods. In [8]-[10] a formal expansion of the scattering amplitude in a series of Mathieu functions has been constructed and in [11]-[13] a formal diagrammatic-like series has been proposed. So far these approaches have had a limited success and did not produce explicit formulas except when the distance between the vortices goes to infinity 13 .

Here we consider the scattering problem on two vortices in the singular gauge with non-trivial boundary conditions along a cut connecting the vortices. To address this problem, we use an approach already put forward in [14] for studying the low-energy spectrum of a charged particle in an harmonic well coupled to two Aharonov-Bohm fluxes of different strength. In the plane a discontinuity has to materialize on any branch cut joining the two vortices due to the non-trivial Aharonov-Bohm phases accumulated by the charged particle moving around one or the other vortex. The positions of the vortices are fixed at $R$ and $-R$ on the $x$-axis, so that the branch cut can be chosen to be the halfcircle of radius $R$ centered at the origin in the lower half-plane (of course the observables, here the modulus squared of the scattering amplitude, should not depend on the choice of a branch cut). As here the total flux is zero, no phase is accumulated at infinity by the particle encircling both vortices. The scattering amplitude is, by definition, a series of scattering amplitudes between incoming and outgoing Hankel partial waves. The non-trivial boundary conditions on the cut lead to an infinite system of equations 


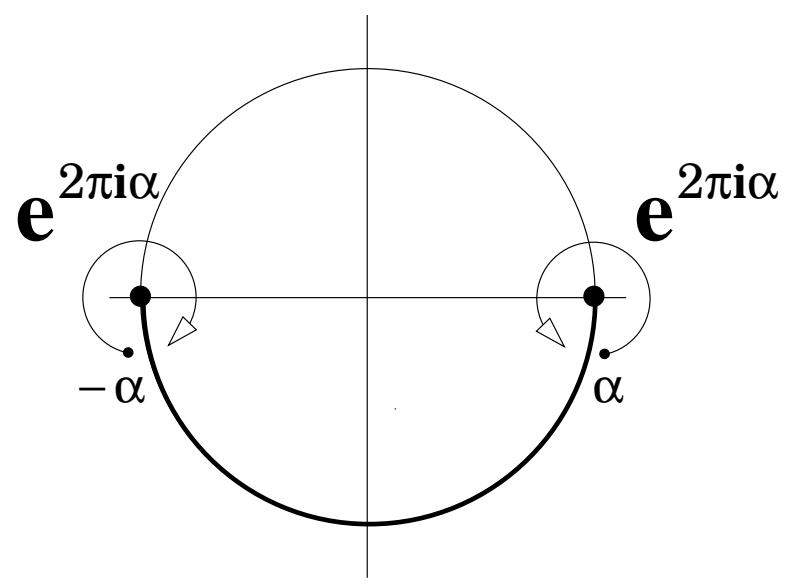

Figure 1. The branch cut in the plane and the boundary conditions for two AharonovBohm vortices with fluxes $\alpha$ and $-\alpha$.

for the expansion coefficients, which are solved numerically. The main part of the paper is devoted to an analytic investigation of two limiting cases. The first is the small flux limit (more precisely, the small $\alpha$ limit, where $\alpha$ is the dimensionless ratio of the flux to the quantum of flux) and the second is the small $R$ limit (in fact the small $k R$ limit, where $k$ is the scattering momentum). In both cases the analytic expressions obtained are in good agreement with the numerical calculations.

The plan of the paper is the following. We start in Section 2 by developing the formalism for the scattering of an incident plane wave making an angle $\theta$ with the $x$ axis. We illustrate in Section 3 the physics at hand with several numerical simulations in various cases of interest. Next, in Section 4 we consider the small $\alpha$ limit and find an exact lowest-order expression for the scattering amplitude. In Section 5 we consider the small $k R$ limit, which allows for some simplifications in the contribution of the relevant partial wave scattering amplitudes. Then in Section 6 we transform the small $k R$ equations into a form which lets us find an exact expression for the scattering amplitude by the Riemann-Hilbert method. In Section 7 we show how the same solution can be obtained and simplified by applying the Riemann-Hilbert method directly to the problem. In Section 8 we outline the main results. A few details of the calculations are given in the Appendix.

\section{General formalism}

We consider two Aharonov-Bohm vortices on a plane at points $\vec{R}$ and $-\vec{R}$, i.e., with polar coordinates $(R, 0)$ and $(R, \pi)$ with fluxes $\alpha$ and $-\alpha$ respectively (see figure 1). We work in the singular gauge, where the electromagnetic potential is removed but the wave function is defined with a cut connecting the two vortices such that on the opposite branches of the cut the wave function and its normal derivative obey the relations

$$
\Psi^{(-)}(R, \phi)=f(\phi) \Psi^{(+)}(R, \phi)
$$


and

$$
\frac{\partial}{\partial r} \Psi^{(-)}(R, \phi)=f(\phi) \frac{\partial}{\partial r} \Psi^{(+)}(R, \phi)
$$

Here and below superscripts $(+)$ and $(-)$ indicate functions outside and inside the circle, respectively, and $f(\phi)$ describes the necessary jump along the cut. In the geometry of figure 1 it can be written in various equivalent forms

$f(\phi)=\left\{\begin{array}{cc}\mathrm{e}^{2 \pi \mathrm{i} \alpha}, & \phi \in I \\ 1, & \phi \notin I\end{array}=1+\left\{\begin{array}{cc}\left(\mathrm{e}^{2 \pi \mathrm{i} \alpha}-1\right), & \phi \in I \\ 0, & \phi \notin I\end{array}=\mathrm{e}^{\pi \mathrm{i} \alpha}\left\{\begin{array}{cc}\mathrm{e}^{\pi \mathrm{i} \alpha}, & \phi \in I \\ \mathrm{e}^{-\pi \mathrm{i} \alpha}, & \phi \notin I\end{array}\right.\right.\right.$

where $I=[\pi \ldots 2 \pi]$ denotes the angular interval of the cut.

Everywhere except the cut the wave function obeys a free Schrödinger equation

$$
\left(\Delta+k^{2}\right) \Psi=0 .
$$

Inside the cut circle the wave function can be expanded into series of regular elementary solutions of (44). We find it convenient to use the Bessel function basis

$$
\Psi^{(-)}(k r, \phi)=\sum_{m=-\infty}^{\infty} c_{m} J_{|m|}(k r) \mathrm{e}^{\mathrm{i} m \phi} .
$$

Let us denote the wave function outside the circle by $\Psi^{(+)}(k r, \phi)$. To implement the boundary conditions (11) and (2), one multiplies both equations by $\mathrm{e}^{\mathrm{i} m \phi}$ and integrates over $\phi$ from 0 to $2 \pi$ for all integer $m$ from $-\infty$ to $\infty$. One concludes that

$J_{|m|}^{\prime}(x) \int_{0}^{2 \pi} \mathrm{e}^{-\mathrm{i} m \phi} \Psi^{(+)}(x, \phi) f(\phi) \mathrm{d} \phi-J_{|m|}(x) \int_{0}^{2 \pi} \mathrm{e}^{-\mathrm{i} m \phi} \frac{\partial}{\partial x}\left(\Psi^{(+)}(x, \phi)\right) f(\phi) \mathrm{d} \phi=0$

where $x=k R$.

The usual way to exploit these conditions (cf. [14]) is to expand $\Psi^{(+)}(r, \phi)$ into a series of Hankel functions provided that the incident wave is fixed:

$$
\Psi_{l}^{(+)}(k r, \phi)=J_{|l|}(k r) \mathrm{e}^{\mathrm{i} l \phi}+\sum_{n=-\infty}^{\infty} t_{n}(l) H_{|n|}^{(1)}(k r) \mathrm{e}^{\mathrm{i} n \phi} .
$$

From (6) it follows that the coefficients $t_{n}(l)$ obey an infinite system of equations

$$
\sum_{n=-\infty}^{\infty}\left(J_{|m|}^{\prime} H_{|n|}^{(1)}-J_{|m|} H_{|n|}^{(1) \prime}\right) A_{m n} t_{n}(l)=-\left(J_{|m|}^{\prime} J_{|l|}-J_{|m|} J_{|l|}^{\prime}\right) A_{m l},
$$

where the $A_{m n}$ 's are the Fourier coefficients of $f(\phi)$ in (3)

$$
A_{m n}=\frac{1}{2 \pi} \int_{0}^{2 \pi} f(\phi) \mathrm{e}^{\mathrm{i}(n-m) \phi} \mathrm{d} \phi=\mathrm{e}^{\mathrm{i} \pi \alpha}\left\{\begin{array}{cc}
\cos \pi \alpha & (m=n) \\
\frac{\sin \pi \alpha}{\pi} \frac{1-(-1)^{n-m}}{n-m} & (m \neq n)
\end{array} .\right.
$$

Here and below, when the argument of Bessel functions is not specified, it is meant to be equal to $k R \equiv x$. It is convenient to rescale the $t_{n}(l)$ 's as

$$
t_{n}(l)=\frac{J_{|l|}(x)}{H_{|n|}^{(1)}(x)} y_{n}(l)
$$


so that the $y_{n}(l)$ 's obey the system of equations

$$
\sum_{n=-\infty}^{\infty}\left(\frac{J_{m}^{\prime}}{J_{m}}-\frac{H_{n}^{(1) \prime}}{H_{n}^{(1)}}\right) A_{m n} y_{n}(l)=-\left(\frac{J_{m}^{\prime}}{J_{m}}-\frac{J_{l}^{\prime}}{J_{l}}\right) A_{m l} .
$$

From figure 1 it is clear that the scattering problem is symmetric with respect to a reflection in the $y$-axis. Namely, if $\Psi(r, \phi)$ is a solution then $\Psi(r, \pi-\phi)$ is also a solution. This symmetry is a consequence of the invariance of the function $f(\phi)$ under the transformation

$$
f(\pi-\phi)=f(\phi) .
$$

It manifests itself in a symmetry of the matrix elements $A_{m n}$

$$
A_{-m,-n}=(-1)^{n-m} A_{m n}
$$

and of the expansion coefficients $y_{n}(l)$

$$
y_{-n}(-l)=(-1)^{n+l} y_{n}(l)
$$

The matrix $A_{m n}$ has two more symmetries. A trivial one corresponds to an integer shift $\alpha \rightarrow 1+\alpha$, which leaves $A_{m n}$ and $y_{n}(l)$ invariant:

$$
y_{n}(l ; \alpha)=y_{n}(l ; 1+\alpha) .
$$

This invariance reflects the well known fact that the Aharonov-Bohm effect depends only on the fractional part of $\alpha$.

The second symmetry is related with the transformation $\alpha \rightarrow-\alpha$. From (9) one concludes that

$$
A_{m n}(-\alpha)=\mathrm{e}^{-2 \pi \mathrm{i} \alpha} A_{-m-n}(\alpha)=(-1)^{m-n} \mathrm{e}^{-2 \pi \mathrm{i} \alpha} A_{m n}(\alpha)
$$

which leads to the symmetry for the coefficients $y_{n}(l)$

$$
y_{n}(l ;-\alpha)=y_{-n}(-l ; \alpha)=(-1)^{n+l} y_{n}(l ; \alpha) .
$$

The knowledge of coefficients $t_{n}(l)$ (or $\left.y_{n}(l)\right)$ determines all other quantities. Of particular interest is the amplitude of scattering of a plane wave on the two vortices. To determine it, one has to find a solution which at large distances from the vortices is the superposition of an incoming plane wave in the direction $\theta$ and a circular symmetric outgoing wave

$$
\Psi^{(+)}(k r, \phi) \underset{r \rightarrow \infty}{\longrightarrow} \mathrm{e}^{\mathrm{i} k r \cos (\theta-\phi)}+\sqrt{\frac{2}{\pi k r}} \mathrm{e}^{\mathrm{i} k r-\mathrm{i} \pi / 4} F(\theta, \phi) .
$$

Expanding the incoming plane wave into a series of Bessel functions (see, e.g., [15])

$$
\mathrm{e}^{\mathrm{i} k r \cos (\theta-\phi)}=\sum_{l=-\infty}^{\infty} \mathrm{i}^{|l|} J_{|l|}(k r) \mathrm{e}^{\mathrm{i} l(\phi-\theta)}
$$

the scattering wave function can be expressed through $\Psi_{l}^{(+)}(k r, \phi)$ defined in (7) as

$$
\Psi^{(+)}(k r, \phi)=\sum_{l=-\infty}^{\infty} \mathrm{i}^{|l|} \Psi_{l}^{(+)}(k r, \phi) \mathrm{e}^{-\mathrm{i} l \theta}=\mathrm{e}^{\mathrm{i} k r \cos (\theta-\phi)}+\Psi^{(\mathrm{ref})}(k r, \phi) .
$$


$\Psi^{(\mathrm{ref})}(k r, \phi)$ is the reflected field given by a series of Hankel functions of the first kind

$$
\Psi^{(\mathrm{ref})}(k r, \phi)=\sum_{n, l=-\infty}^{\infty} \mathrm{i}^{|l|} t_{n}(l) H_{|n|}^{(1)}(k r) \mathrm{e}^{\mathrm{i} n \phi-\mathrm{i} l \theta} .
$$

From the asymptotic behaviour of the Hankel functions (see, e.g., [15])

$$
H_{n}^{(1)}(k r) \underset{r \rightarrow \infty}{\longrightarrow} \sqrt{\frac{2}{\pi k r}} \mathrm{e}^{\mathrm{i}(k r-\pi n / 2-\pi / 4)}
$$

one gets that the scattering amplitude $F(\theta, \phi)$ in (18) is

$$
F(\theta, \phi)=\sum_{n, l=-\infty}^{\infty} \mathrm{i}^{|l|-|n|} t_{n}(l) \mathrm{e}^{\mathrm{i} n \phi-\mathrm{i} l \theta} .
$$

The symmetries (14) and (17) lead to the symmetries of the scattering amplitude

$$
F(\pi-\theta, \pi-\phi)=F(\theta, \phi)
$$

and

$$
F(\theta, \phi ;-\alpha)=F(\theta, \phi ; 1-\alpha)=F(\pi+\theta, \pi+\phi ; \alpha) .
$$

These relations make it possible to restrict the value of flux to $0<\alpha \leq \frac{1}{2}$.

\section{Numerical results}

We proceed to calculate the scattering amplitude numerically, by solving the systems (11) for $y_{n}(l)$, for different values of $l$, and substituting the result into (10) and then (23). From physical considerations it follows that contributions of partial waves with $|l| \ll k R$ have to be small. In the calculations below we restrict ourselves to $|l| \leq 10 k R$ and check that higher waves do not change the results noticeably.

Truncation in $n$ is more complicated. In order to find the coefficients themselves with good precision, one has to retain much higher values of $n$ in the systems (11), $|n| \leq N$ with $N \sim 100$. Even then, the precision attained at technically feasible values of $N$ is not sufficient, and one has to extrapolate the results to $N \rightarrow \infty$.

For a given finite $N$, we truncate the infinite sum in (11) to $n=-N+1, \ldots, N$, so that the system, for a given $l$, consists of $2 N$ equations for as many variables.

It turns out that convergence in $N$ is improved by employing the technique used in [14], discretizing the boundary conditions. The issue at hand is that by choosing $2 N$ coefficients $t_{n}(l)$ in (7) and, respectively, $2 N$ coefficients $c_{m}$ in (5), one can, in general, satisfy the boundary conditions (1)-(2) exactly for no more than $2 N$ discrete values of $\phi$, rather than for any $\phi$. A convenient recourse is to enforce those conditions at $\phi_{k}=\left(k-\frac{1}{2}\right) \frac{\pi}{N}, k=1, \ldots, 2 N$ (these $2 N$ points are distributed uniformly on the circle and avoid as much as possible the locations of the fluxes, where the wave function is singular). Then, integration over $\phi$ in (6) has to be replaced with summation over $\phi_{k}$. It is easy to see that the only change this entails is a modified expression for $A_{m n}$, namely

$$
A_{m n}=\mathrm{e}^{\mathrm{i} \pi \alpha}\left\{\begin{array}{cc}
\cos \pi \alpha & (m=n) \\
\frac{\sin \pi \alpha}{2 N} \frac{1-(-1)^{n-m}}{\sin \frac{(n-m) \pi}{2 N}} & (m \neq n) .
\end{array}\right.
$$


For $N \rightarrow \infty$, one recovers (9), whereas for a given finite $N$, the resulting amplitude is closer to the infinite- $N$ one than the one obtained without this modification.

Convergence in $N$ of the resulting scattering amplitude is slow, due to the usual problem inherent in Aharonov-Bohm or anyon numerics: We are trying to represent a wave function which has a fractional power behaviour at the positions of the vortices (cf. (116)), as an expansion in terms of regular wave functions. The corresponding coefficients fall off slowly. We have found, however, that for all values of $k R, \theta$, and $\alpha$ considered, and for any $\phi \in[-\pi, \pi]$, the $N$ dependence of the amplitude fits an empirical formula

$$
F(\theta, \phi ; N) \simeq F(\theta, \phi)+\frac{a_{1}}{N^{\alpha}}+\frac{a_{2}}{N^{1-\alpha}}+\frac{a_{3}}{N}+\frac{a_{4}}{N^{1+\alpha}}
$$

well, even for rather small $N$. (We have used the values $N=40 \ldots 100$ for the fitting.)

In figures 2, 4 we present the results of numerical calculations for the amplitude of the scattering on two Aharonov-Bohm vortices for fluxes $\alpha=1 / 4,1 / 3$, and $1 / 2$ with $k R=1$ and with incident angles $\theta=0, \pi / 4, \pi / 2$. For clarity, in these figures we count the reflection angle from the direction of the incident wave, i.e., we plot $F(\theta, \theta+\phi)$ instead of $F(\theta, \phi)$.

A notable feature of the results is the absence of symmetry with respect to the reflection $\phi \rightarrow-\phi$. This is in contrast to the standard Aharonov-Bohm scattering, where, despite the fact that chiral symmetry is broken by the presence of the vortex, the amplitude remains symmetric under such reflection. One could have expected the same here for $\theta=0$, when the geometry of the system is just as symmetric with respect to the oncoming wave as it is with a single vortex. However, in general this turns out not to be the case because for the invariance the spacial reflection has to be accompanied by interchanging of the vortices. For $\theta=0$ only the case $\alpha=1 / 2$ is symmetric with respect to inversion $\phi \rightarrow-\phi$ (cf. (24) and (25) ). In general, with positive $\alpha$ the reflected wave is more likely to deviate to the right (negative $\phi$ ). Only for $\theta=\pi / 2$ is the amplitude symmetric, as anticipated (cf. (24) ).

\section{Small $\alpha$ limit}

To find the leading term of the scattering amplitude in the limit $\alpha \rightarrow 0$, it is convenient first to transform the boundary conditions to another form which is of independent interest. Multiplying both sides of ([6]) by $\mathrm{i}^{|m|} \mathrm{e}^{\mathrm{i} m\left(\theta^{\prime}+\pi\right)}$, summing over $m$, and using again the identity (19), one concludes that $\Psi^{(+)}$has to obey

$$
\int_{0}^{2 \pi}\left[\mathrm{e}^{-\mathrm{i} x \cos \left(\theta^{\prime}-\phi\right)} \frac{\partial}{\partial x}\left(\Psi^{(+)}(x, \phi)\right)-\Psi^{(+)}(x, \phi) \frac{\partial}{\partial x}\left(\mathrm{e}^{-\mathrm{i} x \cos \left(\theta^{\prime}-\phi\right)}\right)\right] f(\phi) \mathrm{d} \phi=0 .
$$

This equation can also be obtained by a direct application of the Green identity inside the circle. 
Scattering on two Aharonov-Bohm vortices with opposite fluxes
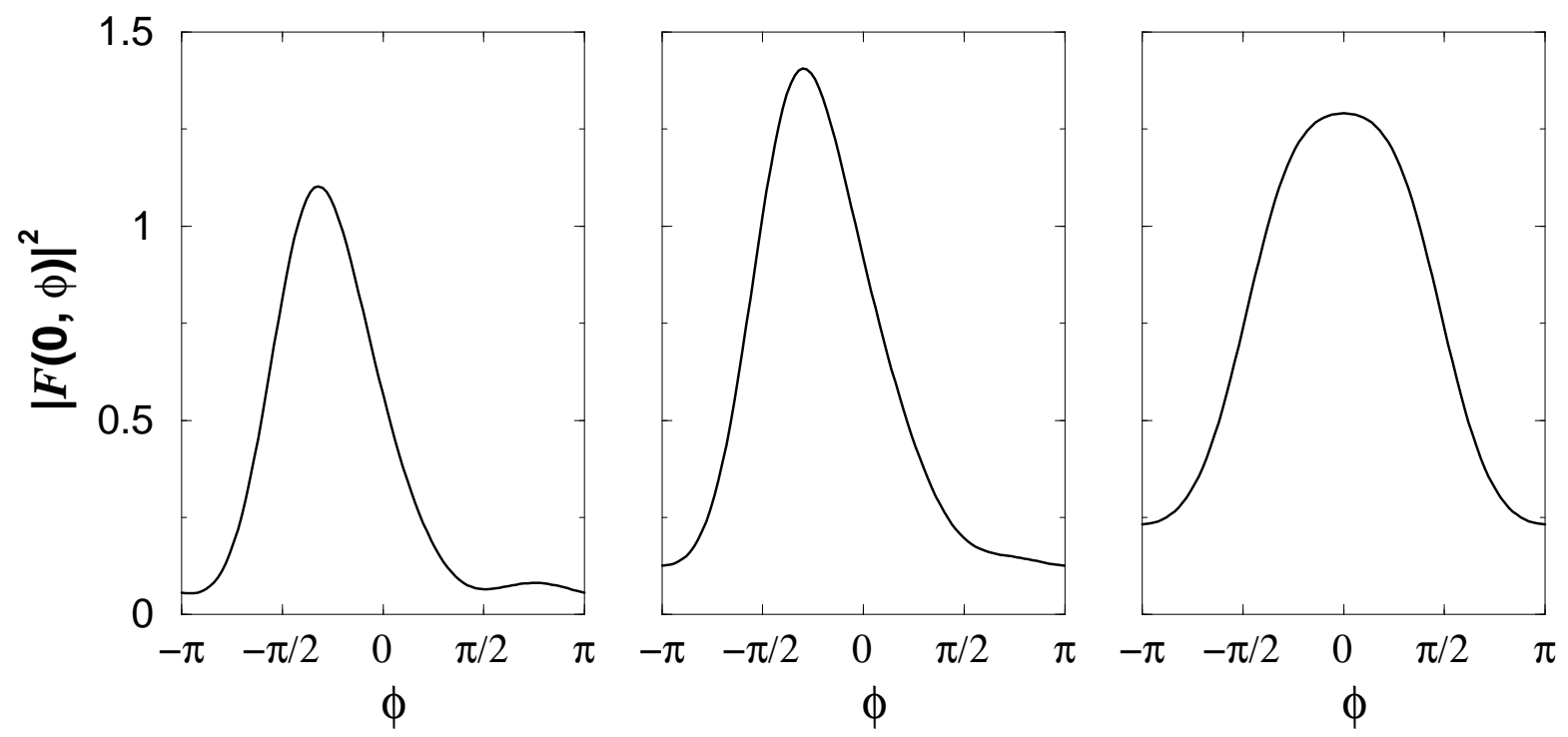

Figure 2. Modulus squared of the scattering amplitude for $k R=1$ and the incident angle $\theta=0$. Left: $\alpha=1 / 4$, center: $\alpha=1 / 3$, right: $\alpha=1 / 2$.
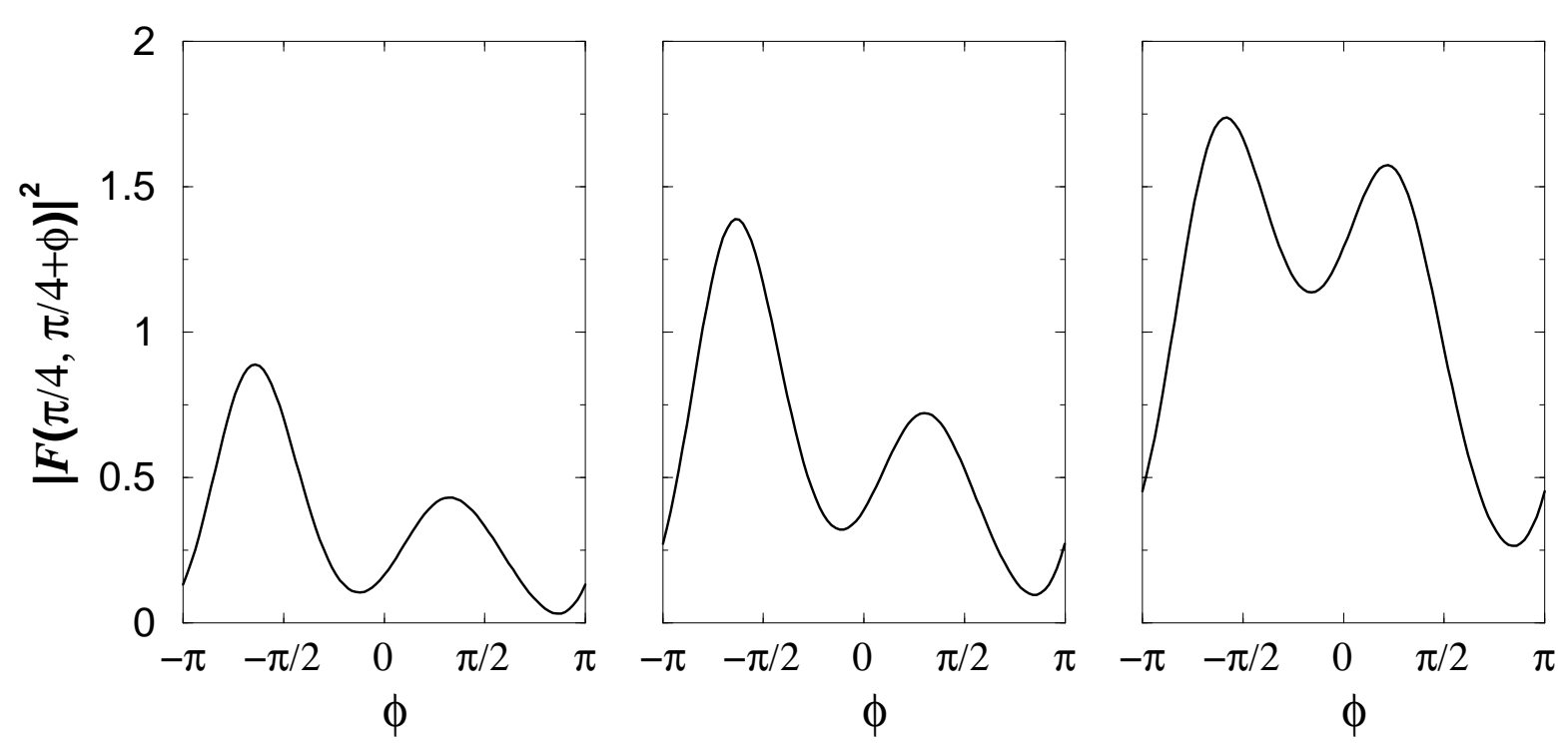

Figure 3. The same as in figure 2 but for the incident angle $\theta=\pi / 4$.

For the pure scattering setup one has to take $\Psi^{(+)}(k r, \phi)$ as in (20). From (28) it follows that the reflected field obeys the equation

$$
\begin{aligned}
\int_{0}^{2 \pi}\left[\mathrm{e}^{-\mathrm{i} x \cos \left(\theta^{\prime}-\phi\right)} \frac{\partial}{\partial x}\left(\Psi^{(\mathrm{ref})}(x, \phi)\right)\right. & \left.-\Psi^{(\mathrm{ref})}(x, \phi) \frac{\partial}{\partial x}\left(\mathrm{e}^{-\mathrm{i} x \cos \left(\theta^{\prime}-\phi\right)}\right)\right] f(\phi) \mathrm{d} \phi \\
& =J\left(\theta, \theta^{\prime}\right)
\end{aligned}
$$

where

$J\left(\theta, \theta^{\prime}\right)=\int_{0}^{2 \pi}\left[\mathrm{e}^{-\mathrm{i} x \cos \left(\theta^{\prime}-\phi\right)} \frac{\partial}{\partial x}\left(\mathrm{e}^{\mathrm{i} x \cos (\theta-\phi)}\right)-\frac{\partial}{\partial x}\left(\mathrm{e}^{-\mathrm{i} x \cos \left(\theta^{\prime}-\phi\right)}\right) \mathrm{e}^{\mathrm{i} x \cos (\theta-\phi)}\right] f(\phi) \mathrm{d} \phi$. 
Scattering on two Aharonov-Bohm vortices with opposite fluxes
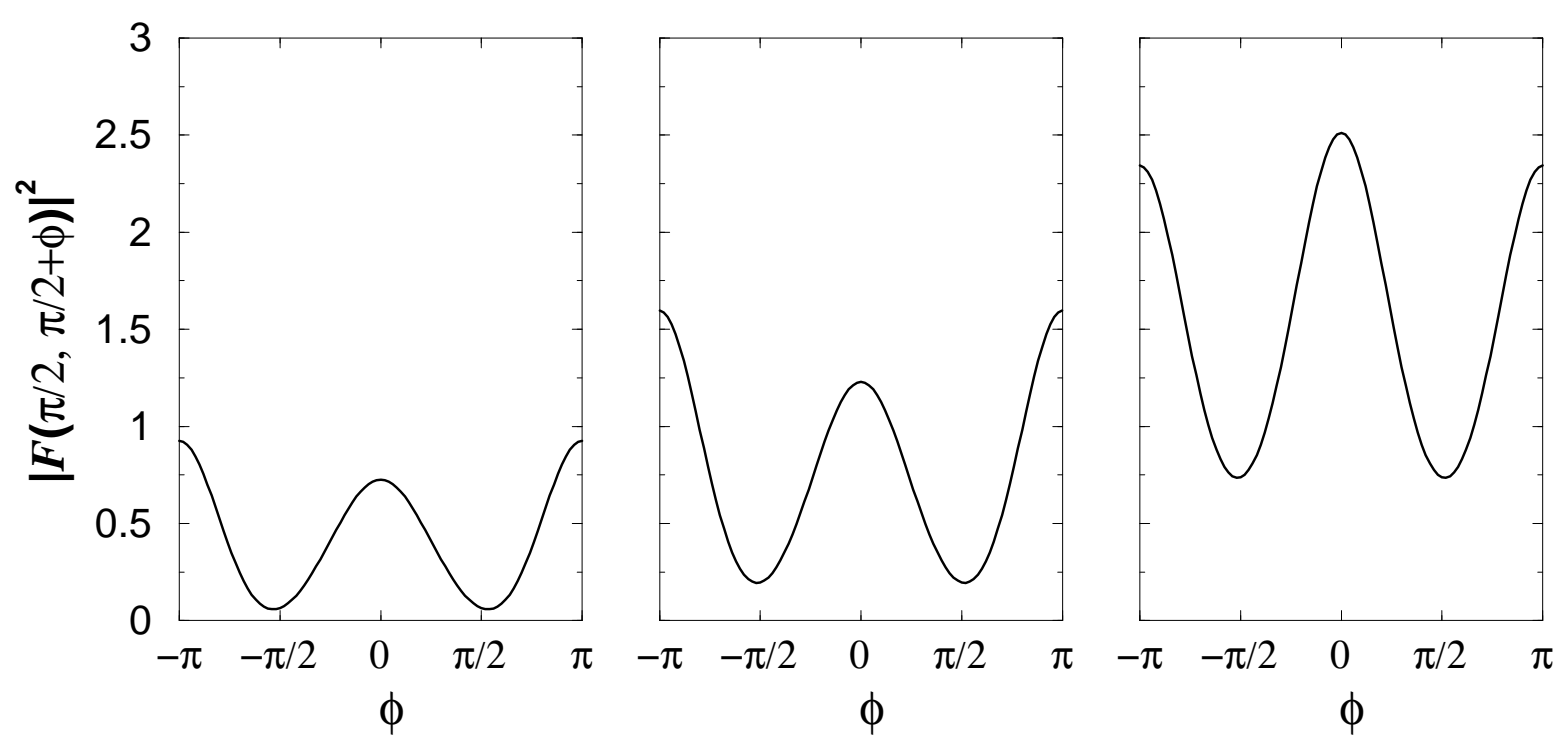

Figure 4. The same as in figure 2 but for but for the incident angle $\theta=\pi / 2$.

The expression in the square brackets is a total derivative:

$$
\begin{aligned}
& \mathrm{e}^{-\mathrm{i} x \cos \left(\theta^{\prime}-\phi\right)} \frac{\partial}{\partial x}\left(\mathrm{e}^{\mathrm{i} x \cos (\theta-\phi)}\right)-\frac{\partial}{\partial x}\left(\mathrm{e}^{-\mathrm{i} x \cos \left(\theta^{\prime}-\phi\right)}\right) \mathrm{e}^{\mathrm{i} x \cos (\theta-\phi)} \\
= & \frac{2 \mathrm{i}}{x} \cot \left(\frac{\theta-\theta^{\prime}}{2}\right) \frac{\partial}{\partial \phi} \exp \left[-2 \mathrm{i} x \sin \left(\frac{\theta+\theta^{\prime}}{2}-\phi\right) \sin \left(\frac{\theta-\theta^{\prime}}{2}\right)\right]
\end{aligned}
$$

so the integration in (29) for $f(\phi)$ given in (3) easily follows as

$$
J\left(\theta, \theta^{\prime}\right)=\frac{4}{x} \mathrm{e}^{\mathrm{i} \pi \alpha} \sin \pi \alpha \cot \frac{\theta-\theta^{\prime}}{2} \sin \left(2 x \sin \frac{\theta-\theta^{\prime}}{2} \sin \frac{\theta+\theta^{\prime}}{2}\right) .
$$

Using the second expression for $f(\phi)$ in (3), (29) can be rewritten as

$$
\begin{aligned}
\Phi\left(\theta, \theta^{\prime}\right) & =-2 \mathrm{e}^{\pi \mathrm{i} \alpha} \sin \pi \alpha \int_{\pi}^{2 \pi}\left[\mathrm{e}^{-\mathrm{i} x \cos \left(\theta^{\prime}-\phi\right)} \frac{\partial}{\partial x} \Psi^{(\mathrm{ref})}(r, \phi)\right. \\
& \left.-\frac{\partial}{\partial x}\left(\mathrm{e}^{-\mathrm{i} x \cos \left(\theta^{\prime}-\phi\right)}\right) \Psi^{(\mathrm{ref})}(r, \phi)\right] \mathrm{d} \phi \\
& +\frac{4}{x} \mathrm{e}^{\mathrm{i} \pi \alpha} \sin \pi \alpha \cot \frac{\theta-\theta^{\prime}}{2} \sin \left(2 x \sin \frac{\theta-\theta^{\prime}}{2} \sin \frac{\theta+\theta^{\prime}}{2}\right)
\end{aligned}
$$

where

$\Phi\left(\theta, \theta^{\prime}\right)=\int_{0}^{2 \pi}\left[\mathrm{e}^{-\mathrm{i} x \cos \left(\theta^{\prime}-\phi\right)} \frac{\partial}{\partial x} \Psi^{(\mathrm{ref})}(r, \phi)-\frac{\partial}{\partial x}\left(\mathrm{e}^{-\mathrm{i} x \cos \left(\theta^{\prime}-\phi\right)}\right) \Psi^{(\mathrm{ref})}(r, \phi)\right] \mathrm{d} \phi$.

It is well known (and easily checked either by the Green representation of the reflected field or by the direct substitution of (21) in (34)) that $\Phi\left(\theta, \theta^{\prime}\right)$ is proportional to the scattering amplitude for the reflected field

$$
\Phi\left(\theta, \theta^{\prime}\right)=\frac{4 \mathrm{i}}{x} F\left(\theta, \theta^{\prime}\right) .
$$



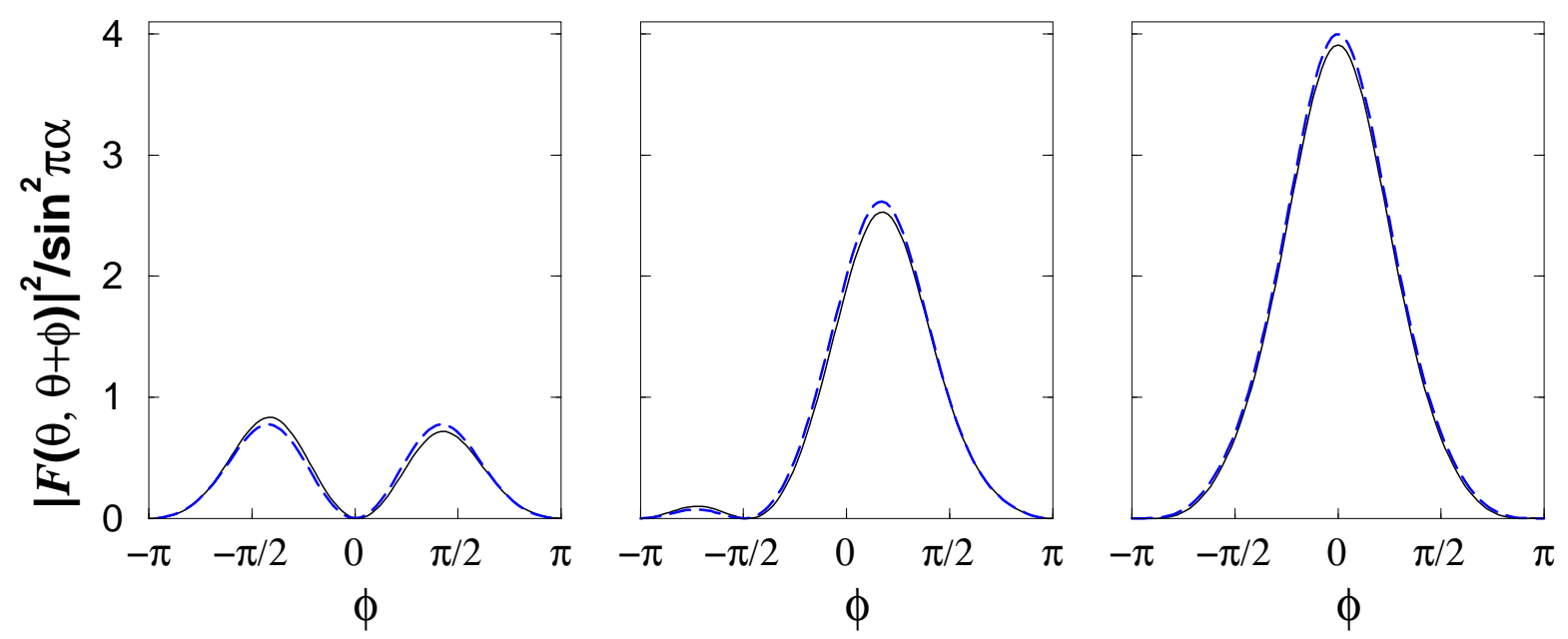

Figure 5. Scattering amplitudes on two vortices with $\alpha=.01$ and $k R=1$ for different incident angle $\theta$ versus the reflection angle $\phi$. Left: $\theta=0$, center: $\theta=\pi / 4$, right: $\theta=\pi / 2$. Solid lines are direct numerical solutions of (11). Dashed lines are calculated from the analytic formula (37).

If one assumes that at small $\alpha$ (hence, small $\sin \pi \alpha$ ) the wave function $\Psi^{(\mathrm{ref})}(r, \phi)$ can be expanded into a series in $\alpha$ as

$$
\Psi^{(\mathrm{ref})}(r, \phi)=\alpha \Psi_{1}(r, \phi)+\alpha^{2} \Psi_{2}(r, \phi)+\ldots
$$

(the zero order term is absent since when $\alpha$ is an integer there is no Aharonov-Bohm scattering) and takes into account that the right-hand side of (33) is proportional to $\sin \pi \alpha$, then the scattering amplitude in the leading order can be approximated by

$$
F\left(\theta, \theta^{\prime}\right) \approx-\mathrm{i} \sin \pi \alpha \cot \frac{\theta-\theta^{\prime}}{2} \sin \left(2 x \sin \frac{\theta-\theta^{\prime}}{2} \sin \frac{\theta+\theta^{\prime}}{2}\right) .
$$

The existence of a term proportional to $N^{-\alpha}$ in the extrapolation formula (27) renders numerical calculations as very small $\alpha$ with $N$ of the order of a few hundred uncertain. Fortunately, the analytical formula (37) for the dominant contribution at small $\alpha$ (37) is regular and does not contain singularities at the vortex positions. This means that the coefficient in front of $N^{-\alpha}$ is, at least, of the second order in $\alpha$. Therefore, at very small $\alpha$ we drop that term from the fit (27).

The results obtained in this manner are presented in figure 5 for different incident angles and compared with (37) for small $\alpha$. For clarity, we divide the scattering amplitude by $\sin \pi \alpha$ and, like in the previous Section, count the reflection angle from the direction of the incident wave. The agreement is quite good and the differences between both curves are of the order of the terms which have been neglected (i.e., $\sin \pi \alpha$ ).

\section{Small $k R$ limit}

Another interesting limit corresponds to the vortices close to each other, so that $k R \ll 1$. In this case it is convenient to use the rescaled variables (11) and to expand all quantities 
Scattering on two Aharonov-Bohm vortices with opposite fluxes

at small $x$. One gets (see, e.g., [15])

$$
\frac{J_{m}^{\prime}(x)}{J_{m}(x)} \underset{x \rightarrow 0}{\longrightarrow} \frac{|m|}{x}, \quad \frac{H_{n}^{(1) \prime}(x)}{H_{n}^{(1)}(x)} \underset{x \rightarrow 0}{\longrightarrow} \frac{-|n|+\delta_{n 0} \rho}{x}
$$

with

$$
\rho=\frac{2 \mathrm{i}}{\pi+2 \mathrm{i}(\ln (x / 2)+\gamma)}
$$

( $\gamma$ is the Euler constant).

In this limit equations (11) take the form

$$
\sum_{n=-\infty}^{\infty}\left(|m|+|n|-\delta_{n 0} \rho\right) A_{m n} y_{n}(l)=-(|m|-|l|) A_{m l}
$$

so that the dependence on $x=k R$ is entirely contained in $\rho$. On the other hand, from (10) it follows that when $x \rightarrow 0, t_{n}(l) \sim x^{|n|+|l|} y_{n}(l)$. Therefore at small $x$ the dominant contribution to the scattering amplitude comes from the $S$-wave amplitude $(l=0)$, more precisely from $y_{0}(0)$, as it is usual for the scattering on small-size objects (see, e.g., [16]). Nevertheless, to determine $y_{0}(0)$, one still has to solve an infinite system of equations (40) with $l=0$ :

$$
\sum_{n=-\infty}^{\infty}\left(|m|+|n|-\delta_{n 0} \rho\right) A_{m n} y_{n}(0)=-|m| A_{m 0} .
$$

The scattering amplitude (23) is dominated at small $x$ by $y_{0}(0)$

$$
F(\theta, \phi) \underset{x \rightarrow 0}{\longrightarrow} t_{0}(0)=\frac{y_{0}(0)}{H_{0}^{(1)}(x)} \approx-\mathrm{i} \frac{\pi}{2} \rho y_{0}(0)
$$

where we used that, at small $x, H_{0}^{(1)}(x) \approx 2 \mathrm{i} /(\pi \rho)$ with $\rho$ given by (39) (see, e.g., [15]).

From (14) it follows that

$$
y_{-n}(0)=(-1)^{n} y_{n}(0)
$$

and one can transform (41) to a system of equations involving the $y_{n}(0)$ 's with nonnegative $n$ only. When $m=0$ one obtains

$$
\cos \pi \alpha \rho y_{0}-\frac{2 \sin \pi \alpha}{\pi} \sum_{n=1}^{\infty}\left(1-(-1)^{n}\right) y_{n}(0)=0 .
$$

For positive $m$, straightforward calculations demonstrate that due to symmetry (43)

$$
\begin{aligned}
& \sum_{n=-\infty, n \neq 0}^{\infty}(m+|n|) A_{m n} y_{n}(0) \\
= & 2 m \sum_{n=1}^{\infty} A_{m n} y_{n}(0)+\frac{\sin \pi \alpha}{\pi}\left(1+(-1)^{m}\right) \sum_{n=1}^{\infty}\left(1-(-1)^{n}\right) y_{n}(0) .
\end{aligned}
$$


Scattering on two Aharonov-Bohm vortices with opposite fluxes

Using (44) and adding the $n=0$ terms, one gets that (41) with positive $m$ is equivalent to

$$
\begin{aligned}
\sum_{n=1}^{\infty} A_{m n} y_{n}(0) & =\frac{\sin \pi \alpha\left(1-(-1)^{m}\right)}{2 \pi m}\left(1+y_{0}(0)\right) \\
& -\left(\frac{\sin \pi \alpha\left(1-(-1)^{m}\right)}{2 \pi m^{2}}+\frac{\cos \pi \alpha\left(1+(-1)^{m}\right)}{4 m}\right) \rho y_{0}(0) .
\end{aligned}
$$

All the dependence on $x=k R$ in this equation is in $\rho$ only.

It is convenient to redefine the variables $y_{n}(0)$ with $n \geq 1$ as

$$
y_{n}(0)=\frac{1}{2} x_{n}\left(1+y_{0}(0)\right)
$$

and to set

$$
x_{0}=1
$$

so that (46) takes the form

$$
\sum_{n=0}^{\infty} A_{m n} x_{n}=\xi f_{m}
$$

where

$$
\xi=\frac{2 \rho y_{0}(0)}{1+y_{0}(0)}
$$

and

$$
f_{m}=-\frac{\sin \pi \alpha\left(1-(-1)^{m}\right)}{2 \pi m^{2}}-\frac{\cos \pi \alpha\left(1+(-1)^{m}\right)}{4 m} .
$$

Similarly (44) becomes

$$
\frac{\pi}{2} \xi \cot \pi \alpha=\sum_{n=1}^{\infty} x_{n}\left(1-(-1)^{n}\right) .
$$

The system (49) is linear, so its solution has the form

$$
x_{n}=a_{n}+b_{n} \xi
$$

where $a_{n}$ and $b_{n}$ depend on $\alpha$ but not on $\rho$ and not on $y_{0}(0)$. From (152) one concludes that $\xi$ has to be determined by

$$
\frac{\pi}{2} \xi \cot \pi \alpha=\sum_{n=1}^{\infty} a_{n}\left(1-(-1)^{n}\right)+\xi \sum_{n=1}^{\infty} b_{n}\left(1-(-1)^{n} .\right.
$$

Solving (54) for $\xi$ one finds

$$
\frac{\rho y_{0}(0)}{1+y_{0}(0)}=-\frac{1}{\beta(\alpha)}
$$

where $\beta(\alpha)$ is real. Therefore

$$
y_{0}(0)=-\frac{1}{1+\beta(\alpha) \rho} .
$$

where the dependence on $k R$ and $\alpha$ has separated. 
From (42) the scattering amplitude in the small $k R$ limit is

$$
F(\theta, \phi) \underset{k R \rightarrow 0}{\longrightarrow}-\frac{\pi}{\pi+2 \mathrm{i}(\ln (k R / 2)+\gamma+\beta(\alpha))} .
$$

It has the standard form of a short-range scattering amplitude (e.g., the scattering on a small disk of radius $R$ with Dirichlet boundary conditions corresponding to $\beta(\alpha)=0$ (see, e.g., [16])).

A simple way of determining $\beta(\alpha)$ numerically is to fix $\rho$ to a given value $\rho_{0}$ (say, $\left.\rho_{0}=0.3\right)$ and to approximate the infinite system (40) by a truncated one

$$
\sum_{n=-N}^{N}\left(|m|+|n|-\delta_{n 0} \rho\right) A_{m n} y_{n}(0)=-|m| A_{m 0}
$$

with $N$ finite ( $m$ is also taken from $-N$ to $N$ ). (58) is solved numerically and yields $y_{0}(0)$ for a given $N$ as a function of $\alpha$, from which $\beta(\alpha)$ follows using (56). The true $\beta(\alpha)$ is then obtained by taking the limit $N \rightarrow \infty$. As the resulting function should have power singularities at the vortex locations (cf. (116) below), the convergence with increasing $N$ is slow, all the more so when $\alpha$ is close to 0 . We found that at large $N$ a good fit of the numerical data involves the same terms as in (27)

$$
y_{\text {fit }} \simeq a_{0}+\frac{a_{1}}{N^{\alpha}}+\frac{a_{2}}{N^{1-\alpha}}+\frac{a_{3}}{N}+\frac{a_{4}}{N^{1+\alpha}} .
$$

For illustration, in figure 6 we present on the left numerical calculations for $\alpha=.2$ and $\rho=.3$ for $N$ from 150 to 550 . The best fit to the data corresponds to (59) with

$$
a_{0} \simeq-2.2688, a_{1} \simeq-.4504, a_{2} \simeq-133.8, a_{3} \simeq 449.3, a_{4} \simeq-552.0 .
$$

On the right of figure 6, the difference between the data and the best fit is presented. It is small $\left(\sim 10^{-6}\right)$, structureless, and can be attributed to random numerical errors, a strong argument for the validity of the fit. Of course, this type of extrapolation is not stable, depends on the form of the extrapolating curve, and the results should be taken with some care.

In figure 7 we present numerical estimates of $\beta(\alpha)$ for a few values of $\alpha$ calculated by the above method together with the exact expression (89) which will be derived in the next Sections.

In figure 8 the scattering amplitudes calculated numerically directly from the main system of equations (11) for $k R=0.01$ and $k R=0.1$ are plotted for $\alpha=1 / 4,1 / 3,1 / 2$ and the incident angle $\theta=0$. (For other incident angles the pictures are similar.) The dashed lines in this figure represent the small $k R$ prediction (57), where we used the following values of $\beta(\alpha)$ computed from the exact formula (89)

$$
\beta\left(\frac{1}{4}\right)=-2 \ln 2, \beta\left(\frac{1}{3}\right)=\ln 2-\frac{3}{2} \ln 3, \beta\left(\frac{1}{2}\right)=-\ln 2 .
$$

The agreement between numerics and the asymptotic expression is good, with differences between them being of the order of $k R$, as expected. 


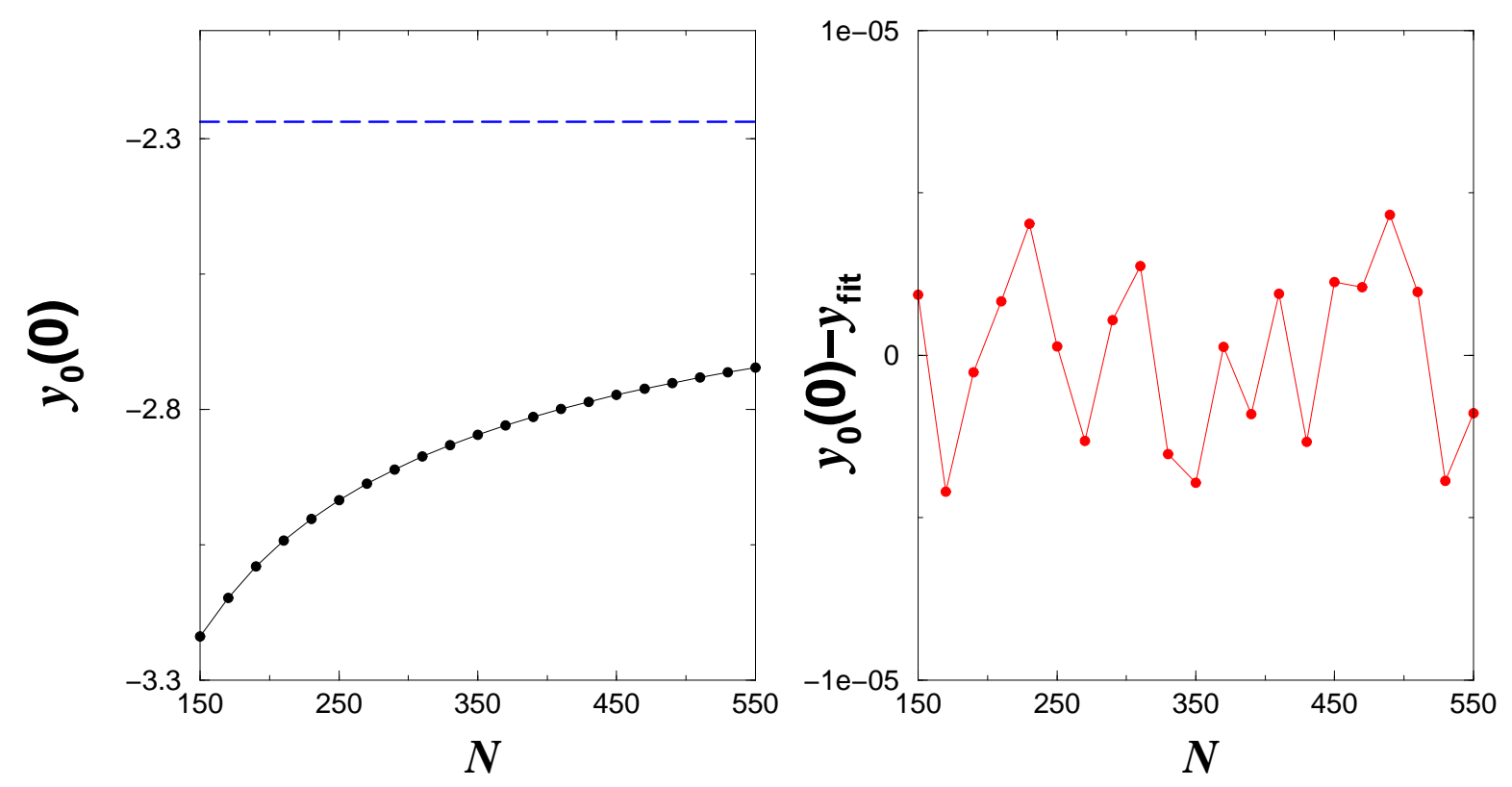

Figure 6. On the left: red circles are the values of $y_{0}(0)$ calculated numerically from (41) with $\alpha=.2$ and $\rho=.3$; the dashed line represents the limiting value of $y_{0}(0)$ (i.e. $a_{0}$ in the fit (59) $)$. On the right: the difference between $y_{0}(0)$ computed numerically at finite $N$ as on the left figure and the fit (59) with fitting coefficients (60). The solid red line serves only to join the points.

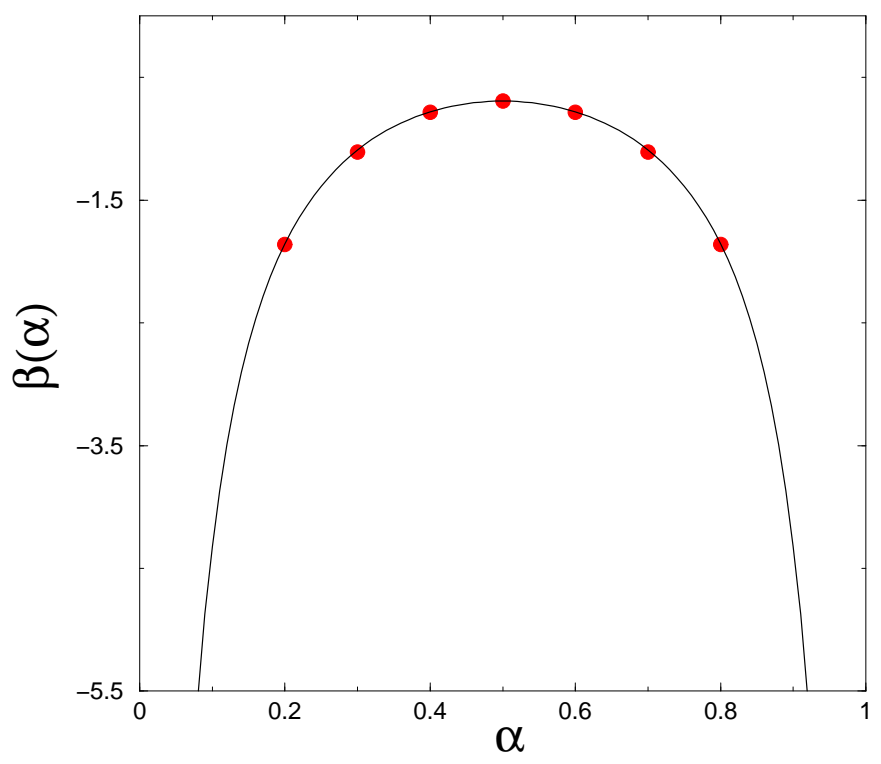

Figure 7. Red circles: $\beta(\alpha)$ calculated numerically; black solid line: exact formula (89). 
Scattering on two Aharonov-Bohm vortices with opposite fluxes
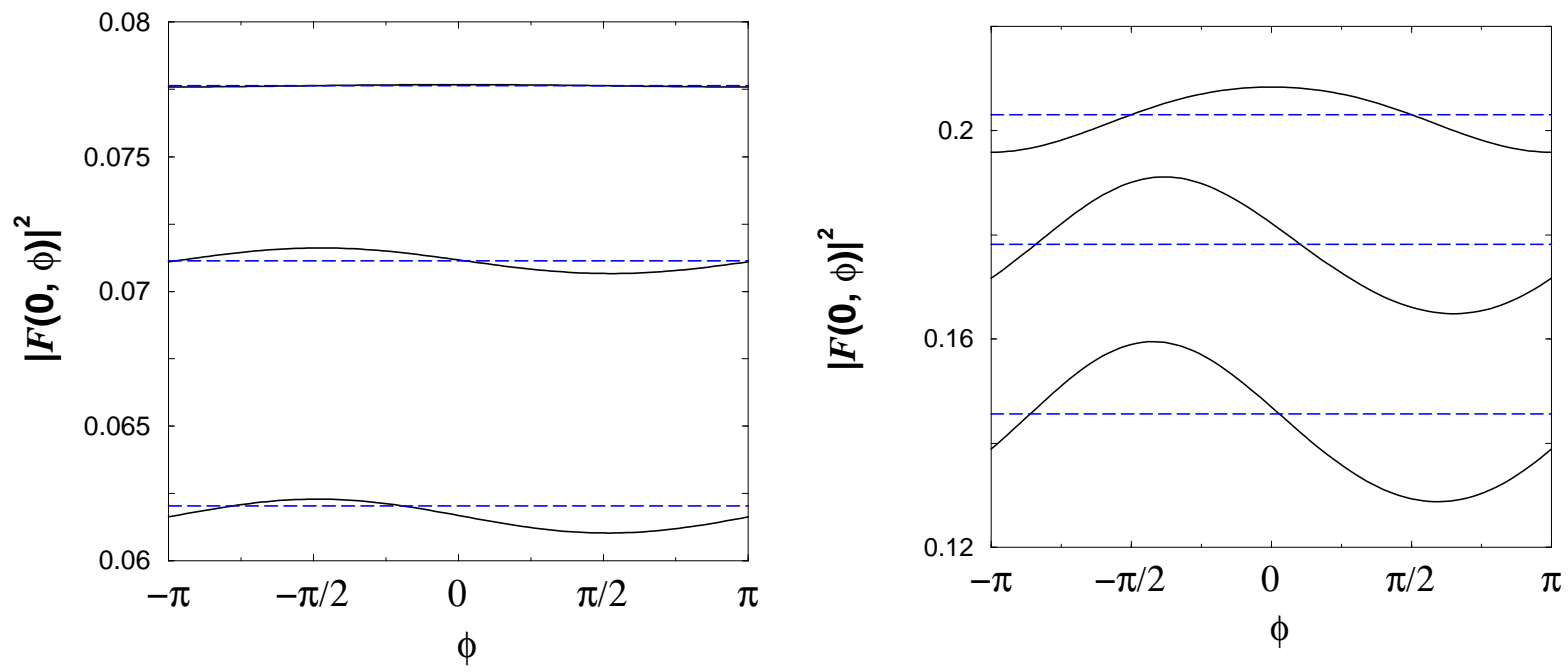

Figure 8. Black solid lines: modulus squared of the scattering amplitude with the incident angle $\theta=0$ and different fluxes. From bottom to top: $\alpha=1 / 4, \alpha=1 / 3$, and $\alpha=1 / 2$. Blue dashed lines are the predictions (57) for the corresponding values of $\alpha$. Left: $k R=0.01$, right: $k R=0.1$.

\section{Exact solution for small $k R$}

The system of equations (49) permits a useful interpretation. Let us define the generating functions of $x_{n}$ and $f_{n}$

$$
\Phi^{(+)}(z)=\sum_{n=0}^{\infty} x_{n} z^{n},
$$

and

$$
F(z)=\sum_{n=1}^{\infty} f_{n} z^{n}
$$

which rewrites, using (51), as

$$
F(z)=\frac{\cos \pi \alpha}{4}(\ln (1-z)+\ln (1+z))-\frac{\sin \pi \alpha}{2 \pi}\left(\operatorname{Li}_{2}(z)-\operatorname{Li}_{2}(-z)\right)
$$

$\left(\operatorname{Li}_{2}(z)=\sum_{n=1}^{\infty} z^{n} / n^{2}\right.$ is Euler's dilogarithm). The normalization (48) leads to $\Phi^{(+)}(0)=$ 1.

In principle, the $x_{n}$ 's are coefficients of an expansion of a function holomorphic outside the circle (see Section 77). It would be more appropriate to use instead of $z=r \mathrm{e}^{\mathrm{i} \phi}$ the variable $1 / \bar{z}=\mathrm{e}^{\mathrm{i} \phi} / r$. To simplify the notations, we use the variable $z$, but retain the superscript $(+)$ in the function (62) to stress that it is related to a function well behaved outside the circle. Equation (49) can then be interpreted as

$$
\Phi^{(+)}\left(\mathrm{e}^{\mathrm{i} \phi}\right) f(\phi)=\Phi^{(-)}\left(\mathrm{e}^{\mathrm{i} \phi}\right)+\xi F\left(\mathrm{e}^{\mathrm{i} \phi}\right)
$$

where $\Phi^{(-)}(z)$ has an expansion in non-positive powers of $z$

$$
\Phi^{(-)}(z)=\sum_{n=0}^{\infty} \frac{d_{n}}{z^{n}} .
$$


Equation (65) is the standard equation for the non-homogeneous Riemann-Hilbert problem (see, e.g., [17]), and its solution can be obtained by the usual methods [17].

First one needs to find the solution of the homogeneous equation

$$
T^{(+)}\left(\mathrm{e}^{\mathrm{i} \phi}\right) f(\phi)=T^{(-)}\left(\mathrm{e}^{\mathrm{i} \phi}\right) .
$$

which can be done by taking its logarithm

$$
\ln T^{(+)}-\ln T^{(-)}=-\ln f(\phi) .
$$

An elementary solution of the last equation is the Cauchy integral [17]

$$
\ln T(z)=-\frac{1}{2 \pi} \int_{0}^{2 \pi} \frac{\ln f(\phi)}{1-z \mathrm{e}^{-\mathrm{i} \phi}} \mathrm{d} \phi .
$$

Here for symmetry reasons we use the third definition of $f(\phi)$ in (3) and again drop the factor $\mathrm{e}^{\mathrm{i} \pi \alpha}$, like in (49):

$$
f(\phi)=\left\{\begin{array}{cc}
\mathrm{e}^{-\mathrm{i} \pi \alpha}, & 0<\phi<\pi \\
\mathrm{e}^{\mathrm{i} \pi \alpha}, & \pi<\phi<2 \pi
\end{array} .\right.
$$

It follows that

$$
T^{(+)}(z)=\left(\frac{1+z}{1-z}\right)^{\alpha}, T^{(-)}(z)=\left(\frac{1+1 / z}{1-1 / z}\right)^{\alpha} .
$$

One easily checks that

$$
T^{( \pm)}\left(\mathrm{e}^{\mathrm{i} \phi}\right)=\left|\cot \frac{\phi}{2}\right|^{\alpha} \begin{cases}\mathrm{e}^{ \pm \mathrm{i} \frac{\pi}{2} \alpha}, & 0<\phi<\pi \\ \mathrm{e}^{\mp \mathrm{i} \frac{\pi}{2} \alpha}, & \pi<\phi<2 \pi\end{cases}
$$

so that (67) is fulfilled.

Using (67), the full non-homogeneous equation (65) can now be rewritten as

$$
\Phi^{(+)}\left(\mathrm{e}^{\mathrm{i} \phi}\right) \frac{T^{(-)}\left(\mathrm{e}^{\mathrm{i} \phi}\right)}{T^{(+)}\left(\mathrm{e}^{\mathrm{i} \phi}\right)}=\Phi^{(-)}\left(\mathrm{e}^{\mathrm{i} \phi}\right)+\xi F\left(\mathrm{e}^{\mathrm{i} \phi}\right)
$$

and therefore

$$
\frac{\Phi^{(+)}\left(\mathrm{e}^{\mathrm{i} \phi}\right)}{T^{(+)}\left(\mathrm{e}^{\mathrm{i} \phi}\right)}-\frac{\Phi^{(-)}\left(\mathrm{e}^{\mathrm{i} \phi}\right)}{T^{(-)}\left(\mathrm{e}^{\mathrm{i} \phi}\right)}=\xi \frac{F\left(\mathrm{e}^{\mathrm{i} \phi}\right)}{T^{(-)}\left(\mathrm{e}^{\mathrm{i} \phi}\right)} .
$$

A solution of (74) is again given by a Cauchy integral

$$
\frac{\Phi(z)}{T(z)}=\frac{\xi}{2 \pi} \int_{0}^{2 \pi} \frac{F\left(\mathrm{e}^{\mathrm{i} \phi}\right)}{T^{(-)}\left(\mathrm{e}^{\mathrm{i} \phi}\right)\left[1-z \mathrm{e}^{-\mathrm{i} \phi}\right]} \mathrm{d} \phi .
$$

Note that since $F(z)$ and $T^{(-)}(z)$ are regular respectively inside and outside the unit circle, the integral (75) cannot be calculated as residues. In the next Section we show that it can be simplified by using a different approach (cf. (113)).

A general solution of (75) has the form [17]

$$
\Phi(z)=\xi T(z) J(z)+T(z) P(z)
$$

where $P(z)$ is a polynomial and

$J(z)=\frac{1}{2 \pi} \int_{0}^{\pi}\left[\mathrm{e}^{\mathrm{i} \pi \alpha / 2} \tan ^{\alpha}(\phi / 2) \frac{F\left(\mathrm{e}^{\mathrm{i} \phi}\right)}{1-z \mathrm{e}^{-\mathrm{i} \phi}}+\mathrm{e}^{-\mathrm{i} \pi \alpha / 2} \cot ^{\alpha}(\phi / 2) \frac{F\left(-\mathrm{e}^{\mathrm{i} \phi}\right)}{1+z \mathrm{e}^{-\mathrm{i} \phi}}\right] \mathrm{d} \phi$. 
Under $\phi \rightarrow \pi-\phi(177)$ has the symmetry

$$
J(\bar{z})=\bar{J}(z) .
$$

In particular, for $z$ real $J(z)$ is real and can be written as

$J(z)=\frac{1}{\pi} \int_{0}^{\pi / 2} \operatorname{Re}\left[\mathrm{e}^{\mathrm{i} \pi \alpha / 2} \tan ^{\alpha}(\phi / 2) \frac{F\left(\mathrm{e}^{\mathrm{i} \phi}\right)}{1-z \mathrm{e}^{-\mathrm{i} \phi}}+\mathrm{e}^{-\mathrm{i} \pi \alpha / 2} \cot ^{\alpha}(\phi / 2) \frac{F\left(-\mathrm{e}^{\mathrm{i} \phi}\right)}{1+z \mathrm{e}^{-\mathrm{i} \phi}}\right] \mathrm{d} \phi$.

Using $\Phi^{(+)}(0)=1$ and the fact that $\Phi^{(-)}(z)$ starts at infinity with a constant we infer from (76) that

$$
\Phi(z)=\xi T(z)[J(z)-J(0)]+T(z) .
$$

Clearly (80) is a solution of (65) and consequently of (49) for all $\xi$ 's. Eq. (52) has yet to be fulfilled. Formally it can be rewritten as

$$
\frac{\pi}{2} \xi \cot \pi \alpha=\lim _{\delta \rightarrow 0}[\Phi(1-\delta)-\Phi(-1)]
$$

where $\Phi(z)$ is the same function as in (62) which is given by (80). ( $\Phi(1)$ in general diverges which explains the necessity of the indicated limit.)

According to (A.11) and (A.20) of Appendix, one has

$$
\Phi(-1)=-\frac{\pi}{4} \cot (\pi \alpha) \xi
$$

and

$$
\Phi(1-\delta)=\left(\frac{2}{\delta}\right)^{\alpha}[\xi(J(1)-J(0))+1]+\frac{\pi}{4} \cot (\pi \alpha) \xi+\mathcal{O}\left(\delta^{1-\alpha}\right)
$$

Comparing these behaviours with (81), we conclude that $\xi$ has to be chosen in such a way that the singularity of (80) at $z=1$ cancels

$$
\xi(J(1)-J(0))+1=0 .
$$

It means that the required solution of (49) is

$$
\Phi(z)=T(z) \frac{J(1)-J(z)}{J(1)-J(0)}
$$

where $T(z)$ and $J(z)$ are defined in (171) and (177) respectively. Expanding $\Phi(z)$ into power of $z$ gives the solution of (49).

One can now calculate all quantities of interest, in particular, $\beta(\alpha)$ in (55)

$$
\beta(\alpha)=2(J(1)-J(0)),
$$

so that using (79) one finally gets

$$
\beta(\alpha)=\frac{2}{\pi} \int_{0}^{\pi / 2} \operatorname{Re}\left[\mathrm{e}^{\mathrm{i} \pi \alpha / 2} \tan ^{\alpha}(\phi / 2) \frac{F\left(\mathrm{e}^{\mathrm{i} \phi}\right)}{\mathrm{e}^{\mathrm{i} \phi}-1}-\mathrm{e}^{-\mathrm{i} \pi \alpha / 2} \cot ^{\alpha}(\phi / 2) \frac{F\left(-\mathrm{e}^{\mathrm{i} \phi}\right)}{\mathrm{e}^{\mathrm{i} \phi}+1}\right] \mathrm{d} \phi .
$$

Note that under $\alpha \rightarrow 1-\alpha, F(z) \rightarrow-F(-z)$, the integrand in the last expression remains symmetric and therefore

$$
\beta(1-\alpha)=\beta(\alpha)
$$

as it follows from (25). 
Using (113), one can prove that when $0<\alpha<1$ (87) is equal to

$$
\beta(\alpha)=\frac{1}{2} \Psi\left(\frac{\alpha}{2}\right)+\frac{1}{2} \Psi\left(\frac{1-\alpha}{2}\right)+\gamma+\ln 4+\frac{\pi}{2 \sin \pi \alpha} .
$$

Here the function $\Psi(z)$ is the logarithmic derivative of the gamma function and $\gamma \equiv-\Psi(1)$ is the Euler constant. $\beta(\alpha)$ calculated numerically from this expression is presented in figure 7 .

\section{Riemann-Hilbert approach}

In the previous Sections we started from a system of equations (41) valid for small $x=k R$ and derived an exact solution using the Riemann-Hilbert method which matches two holomorphic functions regular inside and outside a given contour. Here we demonstrate that the same solution naturally arises from general considerations without explicit transformations of boundary equations.

When $k R \rightarrow 0$, one can ignore in the vicinity of the vortices the $k^{2}$ term in the Schrödinger equation (4) so that the wave function obeys a Laplace equation. It means that it is a sum of a function of the variable $z=r \mathrm{e}^{\mathrm{i} \phi}$ and a function of the variable $\bar{z}=r \mathrm{e}^{-\mathrm{i} \phi}$. Due to the symmetry (14), the $S$-wave function should necessarily have the form

$$
\Psi(r, \phi)=\tilde{\Phi}(z)+\tilde{\Phi}(-\bar{z}) .
$$

Inside the circle the function $\tilde{\Phi}(z)$ has to be regular and can be expanded into a series in $z$

$$
\tilde{\Phi}^{\text {(inside) }}(z)=\sum_{m=0}^{\infty} a_{m}\left(\frac{z}{R}\right)^{m} .
$$

Outside the circle the situation is more complicated. The expansion (7) with the definition (10) states that the outside function has the form

$$
\Psi_{l}^{(+)}(k r, \phi)=J_{|l|}(k r) \mathrm{e}^{\mathrm{i} l \phi}+J_{|l|}(k r) \sum_{n=-\infty}^{\infty} y_{n}(l) \frac{H_{n}^{(1)}(k r)}{H_{n}^{(1)}(k R)} \mathrm{e}^{\mathrm{i} n \phi} .
$$

When $k R \rightarrow 0$ one gets

$$
\Psi_{l}^{(+)}(k r, \phi)=\tilde{\Phi}^{(+)}(z)+\tilde{\Phi}^{(+)}(-\bar{z})+\rho y_{0}(0) \ln \frac{r}{R}
$$

with $\rho$ given in (39). Here

$$
\tilde{\Phi}^{(+)}(z)=\frac{1}{2}\left(1+y_{0}(0)\right)+\sum_{n=1}^{\infty} y_{-n}(0)\left(\frac{R}{z}\right)^{n} .
$$

The logarithmic term appears because of the presence of $H_{0}^{(1)}(k r)$ whose short-distance behaviour requires to add $\ln (r / R)$ to the class of admissible functions. $\ln (r / R)$ is neither analytic nor anti-analytic but can be written as a sum of functions with the same symmetry as in (90)

$$
\ln \left(\frac{r}{R}\right)=\frac{1}{2} \ln \left(-\mathrm{i} \frac{z}{R}\right)+\frac{1}{2} \ln \left(\mathrm{i} \frac{\bar{z}}{R}\right) .
$$


Scattering on two Aharonov-Bohm vortices with opposite fluxes

This argument leads to the conclusion that outside the circle the allowed functions have the form

$$
\tilde{\Phi}^{\text {(outside) }}(z)=\tilde{\Phi}^{(+)}(z)+\frac{1}{2} \rho y_{0}(0) \ln \left(-\mathrm{i} \frac{z}{R}\right) .
$$

The function $\tilde{\Phi}^{(+)}(z)$ is regular outside the circle but the logarithmic function is not. In the splitting (90) it is implicit that if the function $\tilde{\Phi}(z)$ has a cut at $z=|r| \mathrm{e}^{\mathrm{i} \phi_{0}}$ with fixed $\phi_{0}$, it should also have a cut at $z=|r| \mathrm{e}^{\mathrm{i}\left(\pi-\phi_{0}\right)}$. Therefore one has to define $\ln (-\mathrm{i} z)$ on the unit circle $z=\mathrm{e}^{\mathrm{i} \phi}$ with two cuts, the first one from 1 to $\infty$ and the second one from $-\infty$ to -1 ,

$$
\frac{1}{2} \ln \left(-\mathrm{ie}^{\mathrm{i} \phi}\right) \equiv g(\phi)=\frac{\mathrm{i}}{2}\left\{\begin{array}{cc}
\phi-\frac{\pi}{2} & 0<\phi<\pi \\
\phi-\frac{3 \pi}{2} & \pi<\phi<2 \pi
\end{array} .\right.
$$

Now the Aharonov-Bohm boundary conditions (11) are reduced to the condition that the inside and outside functions of variable $z$ (and of $-\bar{z}$ ) are related by

$$
\tilde{\Phi}^{\text {(inside) }}\left(\mathrm{e}^{\mathrm{i} \phi}\right)=f(\phi) \tilde{\Phi}^{\text {(outside) }}\left(\mathrm{e}^{\mathrm{i} \phi}\right) \text {. }
$$

Let us first find two holomorphic functions $F^{(+)}(z)$ and $F^{(-)}(z)$ regular, respectively, outside and inside the circle such that their difference obey

$$
F^{(-)}(z)-F^{(+)}(z)=f(\phi) g(\phi)
$$

with $f(\phi)$ given in (3) and $g(\phi)$ in (97).

The explicit form of $F(z)$ is given again by the Cauchy integral [17]

$$
F(z)=\frac{1}{2 \pi} \oint \frac{f(\phi) g(\phi)}{1-z \mathrm{e}^{-\mathrm{i} \phi}} \mathrm{d} \phi .
$$

Using for $f(\phi)$ the expression (170) one gets

$F(z)=\frac{\mathrm{i}}{4 \pi}\left[\int_{0}^{\pi} \mathrm{e}^{-\mathrm{i} \pi \alpha} \frac{\phi-\pi / 2}{1-z \mathrm{e}^{-\mathrm{i} \phi}} \mathrm{d} \phi+\int_{\pi}^{2 \pi} \mathrm{e}^{\mathrm{i} \pi \alpha} \frac{\phi-3 \pi / 2}{1-z \mathrm{e}^{-\mathrm{i} \phi}} \mathrm{d} \phi\right]=\cos \pi \alpha J_{1}+\sin \pi \alpha J_{2}$

where

$J_{1}=\frac{\mathrm{i}}{4 \pi} \int_{0}^{\pi}(\phi-\pi / 2)\left[\frac{1}{1-z \mathrm{e}^{-\mathrm{i} \phi}}+\frac{1}{1+z \mathrm{e}^{-\mathrm{i} \phi}}\right] \mathrm{d} \phi=\frac{1}{4}[\ln (1+z)+\ln (1-z)]$

and

$J_{2}=\frac{1}{4 \pi} \int_{0}^{\pi}(\phi-\pi / 2)\left[\frac{1}{1-z \mathrm{e}^{-\mathrm{i} \phi}}-\frac{1}{1+z \mathrm{e}^{-\mathrm{i} \phi}}\right] \mathrm{d} \phi=-\frac{1}{2 \pi}\left[\operatorname{Li}_{2}(z)-\operatorname{Li}_{2}(-z)\right]$.

Therefore for $|z| \leq 1$

$$
F^{(-)}(z)=\frac{\cos \pi \alpha}{4}[\ln (1+z)+\ln (1-z)]-\frac{\sin \pi \alpha}{2 \pi}\left[\operatorname{Li}_{2}(z)-\operatorname{Li}_{2}(-z)\right]
$$

which coincides with (64) and for $|z| \geq 1$

$$
F^{(+)}(z)=F^{(-)}(-1 / z) .
$$

Knowing $F(z)$ allows to rewrite (98) as

$$
\tilde{\Phi}^{(-)}\left(\mathrm{e}^{\mathrm{i} \phi}\right)=f(\phi) \tilde{\Phi}^{(+)}\left(\mathrm{e}^{\mathrm{i} \phi}\right)-F^{(+)}\left(\mathrm{e}^{\mathrm{i} \phi}\right) \rho y_{0}(0)
$$


where

$$
\tilde{\Phi}^{(-)}(z)=\tilde{\Phi}^{\text {(inside) }}(z)+F^{(-)}(z) \rho y_{0}(0)
$$

is a function regular inside the circle and $\tilde{\Phi}^{(+)}(z)$ defined in (94) is regular outside the circle.

To transform this equation to the form solved in the previous Section, we note that due to the invariance (12) $f(\pi-\phi)=f(\phi)$ and (106) remains valid under $\phi \rightarrow \pi-\phi$

$$
\tilde{\Phi}^{(-)}\left(-\mathrm{e}^{-\mathrm{i} \phi}\right)=f(\phi) \tilde{\Phi}^{(+)}\left(-\mathrm{e}^{-\mathrm{i} \phi}\right)-F^{(+)}\left(-\mathrm{e}^{-\mathrm{i} \phi}\right) \rho y_{0}(0) .
$$

According to (14) $y_{-n}(0)=(-1)^{n} y_{n}(0)$, so that

$$
\tilde{\Phi}^{(+)}\left(-\mathrm{e}^{-\mathrm{i} \phi}\right)=\frac{1}{2}\left(1+y_{0}(0)\right) \Phi\left(\mathrm{e}^{\mathrm{i} \phi}\right)
$$

with $\Phi(z)$ defined in (62). Denoting $\tilde{\Phi}^{(-)}\left(-\mathrm{e}^{-\mathrm{i} \phi}\right)$ by $\Phi^{(-)}\left(\mathrm{e}^{-\mathrm{i} \phi}\right)$ and taking into account that

$$
F^{(+)}\left(-\mathrm{e}^{-\mathrm{i} \phi}\right)=F^{(-)}\left(\mathrm{e}^{\mathrm{i} \phi}\right) \equiv F\left(\mathrm{e}^{\mathrm{i} \phi}\right)
$$

with $F(z)$ given in (64), we conclude that (108) takes the form

$$
\Phi^{(+)}\left(\mathrm{e}^{\mathrm{i} \phi}\right) f(\phi)=\Phi^{(-)}\left(\mathrm{e}^{\mathrm{i} \phi}\right)+\xi F\left(\mathrm{e}^{\mathrm{i} \phi}\right)
$$

which coincides with (65).

Imposing the condition that the wave functions remain finite at both vortices will fix the value of $y_{0}(0)$ in the same way as above (cf. (84)). Therefore in the end we get the same solution as in the previous Section.

But this approach gives us more than just recalculating the solution by a slightly different method. From (98) together with (96) it is clear that the problem under consideration is equivalent to

$\tilde{\Phi}^{(-)}\left(\mathrm{e}^{\mathrm{i} \phi}\right)=f(\phi) \tilde{\Phi}^{(+)}\left(\mathrm{e}^{\mathrm{i} \phi}\right)+\frac{\rho}{2} y_{0}(0) f(\phi) \ln \left(-\mathrm{ie}^{\mathrm{i} \phi}\right) \equiv f(\phi) \tilde{\Phi}^{(+)}\left(\mathrm{e}^{\mathrm{i} \phi}\right)+\rho y_{0}(0) f(\phi) g(\phi)(11$

where the definition (97) has been used.

This equation is also a non-homogeneous Riemann-Hilbert equation of the same type as (65) with substitution $F\left(\mathrm{e}^{\mathrm{i} \phi}\right) \longrightarrow f(\phi) g(\phi)$. It means that its solution is the same as above but the integral (77) is replaced by

$J(z)=\frac{\mathrm{i}}{4 \pi} \int_{0}^{\pi}\left[\mathrm{e}^{-\mathrm{i} \pi \alpha / 2} \frac{\tan ^{\alpha}(\phi / 2)}{1-z \mathrm{e}^{-\mathrm{i} \phi}}+\mathrm{e}^{\mathrm{i} \pi \alpha / 2} \frac{\cot ^{\alpha}(\phi / 2)}{1+z \mathrm{e}^{-\mathrm{i} \phi}}\right]\left(\phi-\frac{\pi}{2}\right) \mathrm{d} \phi$.

To prove that this expression and (77) are the same we notice that according to (99) and (105)

$$
F\left(\mathrm{e}^{\mathrm{i} \phi}\right)-F\left(-\mathrm{e}^{-\mathrm{i} \phi}\right)=f(\phi) g(\phi)
$$

so $F\left(\mathrm{e}^{\mathrm{i} \phi}\right)-f(\phi) g(\phi)$ contains only negative powers of $\mathrm{e}^{\mathrm{i} \phi}$. When this difference is substituted into the integral (75) the contour can be shifted to infinity and, therefore, the integrals (113) are equal to (77). Other equivalent forms of this integral will be discussed elsewhere. 
A few general comments about the obtained solution are in order. Combining all terms together, we find that in a vicinity of the vortices but outside the cut circle the $S$-wave function in the limit $k R \rightarrow 0$ takes the form

$$
\Psi^{(+)}(\vec{r})=\frac{1}{2}\left(1+y_{0}(0)\right)[\Phi(z)+\Phi(-\bar{z})]+\rho y_{0}(0) \ln |z|
$$

where $\vec{r}$ has coordinates $x$ and $y, z=(x+\mathrm{i} y) / R, \bar{z}=(x-\mathrm{i} y) / R$.

In this expression, $\Phi(z)$ is given by (85) with $z \rightarrow 1 / \bar{z}$. The scattering amplitude $y_{0}(0)$ from the $H_{0}^{(1)}(k r)$ contribution in the expansion (77) is fixed by (56) and (87). That value of $y_{0}(0)$ renders the function $\Phi(z)$ finite at both vortex locations. In fact, as a consequence of (82) and (83), the total wave function (115) vanishes at the vortex positions, as it is the case for the Aharonov-Bohm effect [1]

$$
\Psi(\vec{r}) \sim\left\{\begin{array}{ll}
|\vec{r}-\vec{R}|^{\alpha}, & \vec{r} \text { close to the right vortex with flux } \alpha \\
|\vec{r}+\vec{R}|^{1-\alpha}, & \vec{r} \text { close to the left vortex with flux }-\alpha
\end{array} .\right.
$$

In deriving these limits, we take into account that due to the necessary substitution $z \rightarrow 1 / \bar{z}$, singularities at $z= \pm 1$ in (85) and in (115) are interchanged.

In this Section we use only one of the two Aharonov-Bohm boundary conditions (98), which matches the function on the cut circle, but do not discuss explicitly the second condition (2), which matches the derivative. The reason is that the functions we consider are either analytic or anti-analytic. But for any such function the derivatives with respect to $r$ and $\phi$ are proportional to each other:

$$
\frac{\partial}{\partial r} \Psi(z)=-\frac{\mathrm{i}}{r} \frac{\partial}{\partial \phi} \Psi(z), \quad \frac{\partial}{\partial r} \Psi(\bar{z})=\frac{\mathrm{i}}{r} \frac{\partial}{\partial \phi} \Psi(\bar{z}) .
$$

Therefore, when condition (98) is satisfied and the function $f(\phi)$ is piecewise constant (like in our case) the derivative with respect to $r$ will obey the same condition as the function itself, except for $\delta$-function contributions at the points of discontinuities of $f(\phi)$. One can check that (44) is related with the cancellation of these discontinuities. As has been demonstrated in the previous Section, that equation leads to the vanishing of the wave function at singular points (cf. (116) ), so the $\delta$-functions give no contributions and the derivatives over $r$ automatically obey the correct boundary condition (2).

In [18] it was argued that a solution of the $S$-wave type which remains finite at the two vortex locations cannot exist. But a logarithmic term like the one in (115) was not properly taken into account in that discussion. When ignored (which means setting $\xi=0$ in (85)), only the singular solution $T(z)$ is obtained. The role of the logarithmic term is precisely to remove the singularity at the vortex locations.

\section{Conclusion}

We have considered the scattering problem on two Aharonov-Bohm vortices with opposite fluxes $\alpha$ and $-\alpha$, separated by a distance $2 R$. First, we developed a numerical method for the construction of scattering amplitude. In its simplest formulation it reduces to solving an infinite system of equations (11). The convergence of finite 
approximants is slow due to the singular behaviour of the wave function at the vortex positions (cf. (116)). Then we analytically constructed the dominant contributions to the scattering amplitude in two particular cases: small values of $\alpha$ and small values of $k R$.

In the small $\alpha$ limit, i.e., when $\sin \pi \alpha \rightarrow 0$, the scattering amplitude takes the form

$$
F\left(\theta, \theta^{\prime}\right) \underset{\sin \pi \alpha \rightarrow 0}{\longrightarrow}-\mathrm{i} \sin \pi \alpha \cot \frac{\theta-\theta^{\prime}}{2} \sin \left(2 x \sin \frac{\theta-\theta^{\prime}}{2} \sin \frac{\theta+\theta^{\prime}}{2}\right)
$$

where $\theta$ and $\theta^{\prime}$ are respectively the incident and reflection angles.

In the small $k R$ limit, the solution has been obtained by the Riemann-Hilbert method in its simplest setting. The dominant contribution is given by the $S$-wave scattering amplitude, which has the form

$$
F\left(\theta, \theta^{\prime}\right) \underset{k R \rightarrow 0}{\longrightarrow}-\frac{\pi}{\pi+2 \mathrm{i}(\ln (k R / 2)+\gamma+\beta(\alpha))}
$$

where $\beta(\alpha)$ is

$$
\beta(\alpha)=\frac{1}{2} \Psi\left(\frac{\alpha}{2}\right)+\frac{1}{2} \Psi\left(\frac{1-\alpha}{2}\right)+\gamma+\ln 4+\frac{\pi}{2 \sin \pi \alpha}
$$

with $\Psi(z)$ being the logarithmic derivative of the gamma function.

The same method permits also to find the behaviour of the $S$-wave function in the vicinity of the vortices. Denoting this wave function as

$$
\Psi_{0}^{(+)}(r, \phi)=J_{0}(k r)+J_{0}(k R) \sum_{n=-\infty}^{\infty} \frac{y_{n}(0)}{H_{n}^{(1)}(k R)} H_{n}^{(1)}(k r) \mathrm{e}^{\mathrm{i} n \phi}
$$

we found that when $k R \rightarrow 0$

$$
y_{0}(0)=-\frac{1}{1+\beta(\alpha) \rho}
$$

where

$$
\rho=\frac{2 \mathrm{i}}{\pi+2 \mathrm{i}(\ln (k R / 2)+\gamma)} .
$$

The generating function of $y_{n}(0)=y_{-n}(0)$ in this limit takes the form

$$
\sum_{n=0}^{\infty} y_{n}(0) z^{n}=\frac{1+y_{0}}{2} \Phi(z)-\frac{1}{2} \text {. }
$$

Here

$$
\Phi(z)=\frac{2}{\beta(\alpha)}\left(\frac{1+z}{1-z}\right)^{\alpha}[J(1)-J(z)]
$$

and

$$
J(z)=\frac{\mathrm{i}}{4 \pi} \int_{0}^{\pi}\left[\mathrm{e}^{-\mathrm{i} \pi \alpha / 2} \frac{\tan ^{\alpha}(\phi / 2)}{1-z \mathrm{e}^{-\mathrm{i} \phi}}+\mathrm{e}^{\mathrm{i} \pi \alpha / 2} \frac{\cot ^{\alpha}(\phi / 2)}{1+z \mathrm{e}^{-\mathrm{i} \phi}}\right]\left(\phi-\frac{\pi}{2}\right) \mathrm{d} \phi .
$$

A discussion of other partial waves in the limit $k R \rightarrow 0$ and of related questions in the general case of two Aharonov-Bohm vortices with arbitrary fluxes will be given elsewhere. The exact solution for the scattering amplitude with $k R$ finite remains an open challenge. 
Scattering on two Aharonov-Bohm vortices with opposite fluxes

\section{Appendix A. Limiting values}

To calculate the behaviour of the solution (80) at $z \rightarrow 1$ and $z \rightarrow-1$, one has to investigate the corresponding limits for the integral (179). When $z=-1+\delta$ and $\delta \rightarrow 0$, the second term in (77) is formally diverging. This divergence appears at small $\phi$ and can be isolated by splitting the integral (79) into two parts:

$$
\begin{aligned}
J(-1+\delta) & \equiv \frac{1}{\pi} \int_{0}^{\pi / 2} \operatorname{Re}\left[\mathrm{e}^{\mathrm{i} \pi \alpha / 2} \tan ^{\alpha}(\phi / 2) \frac{F\left(\mathrm{e}^{\mathrm{i} \phi}\right)}{1-(-1+\delta) \mathrm{e}^{-\mathrm{i} \phi}}\right. \\
& \left.+\mathrm{e}^{-\mathrm{i} \pi \alpha / 2} \cot ^{\alpha}(\phi / 2) \frac{F\left(-\mathrm{e}^{\mathrm{i} \phi}\right)}{1+(-1+\delta) \mathrm{e}^{-\mathrm{i} \phi}}\right] \mathrm{d} \phi \\
& =\frac{1}{\pi} \int_{0}^{\pi / 2} \operatorname{Re}\left[\mathrm{e}^{-\mathrm{i} \pi \alpha / 2} \cot ^{\alpha}(\phi / 2) \frac{F\left(-\mathrm{e}^{\mathrm{i} \phi}\right)}{\mathrm{i}(\phi-\mathrm{i} \delta)}\right] \mathrm{d} \phi+\mathcal{O}(1) .
\end{aligned}
$$

Changing the variable in the last integral $\phi \rightarrow t \delta$ gives

$$
J(-1+\delta) \underset{\delta \rightarrow 0}{\longrightarrow} W(\delta)+\mathcal{O}(1)
$$

where

$$
W(\delta)=\frac{1}{\pi} \int_{0}^{\pi /(2 \delta)} \operatorname{Re}\left[\mathrm{e}^{-\mathrm{i} \pi \alpha / 2} \cot ^{\alpha}(\delta t / 2) \frac{F\left(-\mathrm{e}^{\mathrm{i} \delta t}\right)}{\mathrm{i}(t-\mathrm{i})}\right] \mathrm{d} t .
$$

From known expressions

$$
\operatorname{Li}_{2}(1)=\frac{\pi^{2}}{6}, \quad \operatorname{Li}_{2}(-1)=-\frac{\pi^{2}}{12}
$$

it follows that the function $F\left(-\mathrm{e}^{\mathrm{i} \phi}\right)$ has the following expansion

$$
F\left(-\mathrm{e}^{\mathrm{i} \phi}\right) \underset{\phi \rightarrow 0}{\longrightarrow} \frac{1}{4} \cos \pi \alpha\left[\ln 2 \phi-\mathrm{i} \frac{\pi}{2}\right]+\frac{\pi}{8} \sin \pi \alpha .
$$

Finally, one has in the limit $\delta \rightarrow 0$

$W(\delta)=\left(\frac{2}{\delta}\right)^{\alpha} \operatorname{Re} \int_{0}^{\infty} \frac{\mathrm{e}^{-\mathrm{i} \pi \alpha / 2}}{\mathrm{i} \pi t^{\alpha}(t-\mathrm{i})}\left[\frac{1}{4} \cos \pi \alpha\left[\ln (2 \delta t)+\mathrm{i} \frac{\pi}{2}\right]+\frac{\pi}{8} \sin \pi \alpha\right] \mathrm{d} t$.

The first integral in this expression can be calculated by contour integration

$$
\int_{0}^{\infty} \frac{\mathrm{d} t}{t^{\alpha}(t-\mathrm{i})}=\frac{\pi}{\sin \pi \alpha} \mathrm{e}^{\mathrm{i} \pi \alpha / 2}
$$

and the second one by taking the derivative with respect to $\alpha$ of this result

$$
\int_{0}^{\infty} \frac{\mathrm{d} t \ln t}{t^{\alpha}(t-\mathrm{i})}=\frac{\pi^{2}}{\sin \pi \alpha} \mathrm{e}^{\mathrm{i} \pi \alpha / 2}\left(-\frac{\mathrm{i}}{2}+\cot \pi \alpha\right) .
$$

Substituting these expressions into (A.6) and taking the real part, one obtains

$$
W(\delta)=-\left(\frac{2}{\delta}\right)^{\alpha} \frac{\pi \cot (\pi \alpha)}{4}
$$

and

$$
J(-1+\delta) \underset{\delta \rightarrow 0}{\longrightarrow}-\left(\frac{2}{\delta}\right)^{\alpha} \frac{\pi \cot (\pi \alpha)}{4}+\mathcal{O}(1)
$$


Scattering on two Aharonov-Bohm vortices with opposite fluxes

Because $T(-1+\delta) \rightarrow(\delta / 2)^{\alpha}$, from (80) it follows that

$$
\Phi(-1+\delta)=-\frac{\pi}{4} \cot (\pi \alpha) \xi+\mathcal{O}\left(\delta^{\alpha}\right) .
$$

The behaviour of $J(z)$ close to $z=1$ can be obtained by a similar method. The difference is that in this case the value $J(1)$ is finite but due to the singularity of $T(z)$ at $z=1$ we also need to know the correction term. One has

$$
\begin{aligned}
J(1-\delta) & =\frac{1}{\pi} \operatorname{Re} \int_{0}^{\pi / 2}\left[\mathrm{e}^{\mathrm{i} \pi \alpha / 2} \tan ^{\alpha}(\phi / 2) \frac{F\left(\mathrm{e}^{\mathrm{i} \phi}\right)}{1-(1-\delta) \mathrm{e}^{-\mathrm{i} \phi}}\right. \\
& \left.+\mathrm{e}^{-\mathrm{i} \pi \alpha / 2} \cot ^{\alpha}(\phi / 2) \frac{F\left(-\mathrm{e}^{\mathrm{i} \phi}\right)}{1+(1-\delta) \mathrm{e}^{-\mathrm{i} \phi}}\right] \mathrm{d} \phi
\end{aligned}
$$

First we split this integral into two parts as follows

$$
\int_{0}^{\pi / 2} \ldots \mathrm{d} \phi=\int_{0}^{\epsilon} \ldots \mathrm{d} \phi+\int_{\epsilon}^{\pi / 2} \ldots \mathrm{d} \phi
$$

where $\delta \ll \epsilon \ll 1$.

In the first integral, the first term dominates at small $\phi$, and

$$
J(1-\delta) \underset{\delta \rightarrow 0}{\longrightarrow} J(1)+V(\delta)
$$

where

$$
V(\delta)=\frac{1}{\pi} \operatorname{Re} \int_{0}^{\epsilon} \mathrm{e}^{\mathrm{i} \pi \alpha / 2} \tan ^{\alpha}(\phi / 2) F\left(\mathrm{e}^{\mathrm{i} \phi}\right)\left(\frac{1}{\mathrm{i}(\phi-\mathrm{i} \delta)}-\frac{1}{\mathrm{i} \phi}\right) \mathrm{d} \phi .
$$

The second term in the parentheses appears when the splitting (A.13) is applied for the calculation of $J(1)$.

Using an expansion similar to (A.5)

$$
F\left(\mathrm{e}^{\mathrm{i} \phi}\right) \underset{\phi \rightarrow 0}{\longrightarrow} \frac{1}{4} \cos \pi \alpha\left[\ln 2 \phi-\mathrm{i} \frac{\pi}{2}\right]-\frac{\pi}{8} \sin \pi \alpha
$$

and rescaling the variable $\phi=t \delta$, one concludes that

$V(\delta)=\left(\frac{\delta}{2}\right)^{\alpha} \operatorname{Re} \int_{0}^{\infty} \mathrm{e}^{\mathrm{i} \pi \alpha / 2} \frac{t^{\alpha}}{\pi t(t-\mathrm{i})}\left[\frac{1}{4} \cos \pi \alpha\left[\ln (2 t \delta)-\mathrm{i} \frac{\pi}{2}\right]-\frac{\pi}{8} \sin \pi \alpha\right] \mathrm{d} t$.

The remaining integrals are calculated as above:

$$
\int_{0}^{\infty} \frac{t^{\alpha}}{t(t-\mathrm{i})} \mathrm{d} t=\frac{\mathrm{i} \pi}{\sin \pi \alpha} \mathrm{e}^{-\mathrm{i} \pi \alpha / 2}, \int_{0}^{\infty} \frac{t^{\alpha} \ln t}{t(t-\mathrm{i})} \mathrm{d} t=\frac{\mathrm{i} \pi^{2}}{\sin \pi \alpha} \mathrm{e}^{-\mathrm{i} \pi \alpha / 2}\left(-\frac{\mathrm{i}}{2}-\cot \pi \alpha\right) .
$$

Using these values, one gets

$$
V(\delta)=\left(\frac{\delta}{2}\right)^{\alpha} \frac{\pi}{4} \cot \pi \alpha
$$

As $T(1-\delta) \underset{\delta \rightarrow 0}{\longrightarrow}(2 / \delta)^{\alpha}$ we conclude that

$$
\Phi(1-\delta)=\left(\frac{2}{\delta}\right)^{\alpha}[\xi(J(1)-J(0))+1]+\frac{\pi}{4} \cot (\pi \alpha) \xi+\mathcal{O}\left(\delta^{1-\alpha}\right) .
$$


Scattering on two Aharonov-Bohm vortices with opposite fluxes

\section{References}

[1] Aharonov Y and Bohm D 1959 Significance of electromagnetic potentials in the quantum theory, Phys. Rev. 115485

[2] Tonomura A, Osakabe N, Matsuda T, Kawasaki T, Endo J, Yano S and Yamada H (1986) Evidence for Aharonov-Bohm effect with magnetic field completely shielded from electron wave Phys. Rev. Lett. 56792

[3] Comtet A, Mashkevich S and Ouvry S 1995 Magnetic moment and perturbation theory with singular magnetic fields Phys. Rev. D 522594

[4] Ruijsenaars S N M 1983 The Aharonov Bohm effect and the scattering theory Ann. Phys., NY 1461

[5] Peshkin M and Tonomura A 1989 The Aharonov-Bohm effect (Lecture Notes in Physics vol 340) (Springer-Verlag, Berlin)

[6] Franchini F and Goldhaber A S 2008 Aharonov-Bohm effect with many vortices Phys. Scr. 78 065002

[7] Desbois J, Furtlehner C and Ouvry S 1995 Random magnetic impurities and the Landau problem Nucl. Phys. B 453759

[8] Zhi-Yu Gu and Shang-Wu Qian 1988 Aharonov-Bohm scattering on parallel flux lines of the same magnitude J. Phys. A: Math. Gen. 21 2573; ibid 1990 Corrigendum J. Phys. A: Math. Gen. 23 4183

[9] Zhi-Yu Gu and Shang-Wu Qian 1990 Aharonov-Bohm scattering on parallel flux lines of the same magnetic flux J. Phys. A: Math. Gen. 234127

[10] Zhi-Yu Gu and Shang-Wu Qian 1990 Aharonov-Bohm scattering on two antiparallel flux lines of the same magnitude - without return flux 264441

[11] Šťovíček P 1989 The Green function for the two-solenoid Aharonov-Bohm effect Phys. Lett. A 142 5

[12] Šťovíček P 1991 Scattering matrix for the two-solenoid Aharonov-Bohm effect Phys. Lett. A 161 13

[13] Šťovíček P 1993 Scattering on two Aharonov-Bohm solenoids Phys. Rev. A 483987

[14] Mashkevich S, Myrheim J and Ouvry S 2004 Quantum mechanics of a particle with two magnetic impurities Phys. Rev. Lett. A 33041

[15] Erdélyi (ed) 1953 Higher transcendental functions vol II (McGraw-Hill, New York, Toronto, London)

[16] Albeverio S, Gesztesy F, Høegh-Krohn R and Holden H 1988 Solvable models in quantum mechanics (Springer, New York, Berlin)

[17] Muskhelishvili N I 1953 Singular integral equations: Boundary problems of function theory and their application to mathematical physics (P. Noordhoff, Grotingen-Hollland)

[18] Nambu Y 2000 The Aharonov-Bohm problem revisited Nucl. Phys. B 579590 